BIJDRAGEN TOT DE KENNIS DER FAUNA VAN CURAÇAO.

Resultaten eener Reis van Dr. C. J. VAN DER HORST in 1920.

\title{
ACTINIARIEN, ZOANTHARIEN UND CERIANTHARIEN VON CURAÇAO
}

\author{
VON \\ Professor Dr. F. PAX (Breslau). \\ (Mit 2 Karten, 1 Tafel und 22 Abbildungen im Text).
}

Über die Actiniarien, Zoantharien und Ceriantharien von Niederländisch-Westindien war bisher fast nichts bekannt, auch enthalten die holländischen Museen nur sehr wenig Material aus diesem Gebiete. Aus Curaçao lagen, soweit ich die Literatur überblicke, bisher überhaupt noch keine Funde vor. Es ist daher mit besonderer Freude zu begrüssen, dass Herr Dr. VAN DER HORST (Amsterdam) einen zweimonatigen Aufenthalt auf Curaçao im Jahre 1920 dazu benützt hat, eine umfangreiche Sammlung von Actiniarien, Zoantharien und Ceriantharien anzulegen, deren Bestimmung ich um so lieber übernommen habe, als es sich fast ausnahmslos um vorzüglich erhaltene Stücke handelt. Die Sammlung VAN DER HORST umfasst insgesamt 27 Arten, von denen 19 auf die Actiniarien, 7 auf die Zoantharien und 1 auf die Ceriantharien entfallen. Wenn man berücksichtigt, dass von den Bahama-Inseln, auf denen im Sommer 1887 MC MURRICH, einige Jahre später J. I. NORThROP, 1893 die von der Universität Iowa ausgerüstete Bahama-Expedition sammelte und in den Jahren 1908 und 1909 der schwedische Zoologe RosÉN tätig war, nach WATzLS (1922) Zusammenstellung nur 23 Arten Seeanemonen bekannt sind, wenn man sich ferner vergegenwärtigt, dass durch die langjährigen Studien DuERDENs, dem die vorzüglichen Hilfsmittel des Institute of Jamaica zur Verfügung standen, etwa 38 Arten-Actiniarien, Zoantharien und Ceriantharien aus den Gewässern um Jamaica nachgewiesen werden konnten, und wenn man schliesslich bedenkt, dass die im Jahre 1907 von KÖKENTHAL und HARTMEYER unternommene Forschungsreise nach Westindien, die in erster Linie der Untersuchung der Anthozoenfauna der Antillen diente, eine Ausbeute von 26 Species der drei erwähnten Korallengruppen lieferte, wird man nicht umhin können, die Sammlung vAN DER HORST als ungewöhnlich reichhaltig zu bezeichnen. Alle Exemplare dieser Kollektion sind nach Angabe der Fundortzettel zwischen dem 3. April und dem 26. Mai 1920, also innerhalb eines verhältnismässig kurzen Zeitraumes gesammelt worden, auch fehlen Tiefwasserformen vollständig, da dem Sammler die für den Fang dieser Tiere erforderlichen Geräte nicht zur Verfügung standen. Trotzdem dürfen wir nach meinem Dafürhalten keineswegs von dem günstigen Ergebnis einer nur kurzen Sammeltätigkeit auf einen besonderen Artenreichtum der Anthozoenfauna von Curaçao schliessen. Vielmehr bestätigt eine Durchsicht der Sammlung VAN DER HORST lediglich die alte Erfahrung, dass die gründliche Untersuchung eines räumlich begrenzten Geländes bessere Resultate liefert als die Entnahme zahlreicher Stichproben an möglichst vielen Standorten.

Das mir vorliegende Material wurde an der Südküste der Insel Curaçao gesammelt, hauptsächlich in der Caracas-Bai und einer als "Spaansch Water” bezeichneten, weit in das Land eingreifenden und mit der offenen See durch einen schmalen Kanal in Verbindung stehenden Bucht. Einige wenige Stücke entstammen auch dem „Spaansche Haven”, der „Boca labadera” sowie der Westspitze der Insel („Westpunt”). Wie mir Herr Dr. vAN DER HORST mitteilt, ist das Wasser der Caracas-Bai völlig klar, während das Spaansch Water regelmässig starke Trübungen aufweist. Dieser Unterschied der 
Standortsbedingungen macht sich nach den Beobachtungen des Sammlers in der Zusammensetzung der Madreporàrienfauna deutlich bemerkbar. Auch die von mir bearbeiteten Korallengruppen verhalten sich hinsichtlich der Besiedlung der beiden Standorte verschieden:

\begin{tabular}{|c|c|c|c|c|c|}
\hline Species. & $\begin{array}{c}\text { Caracas- } \\
\text { Bai. }\end{array}$ & $\begin{array}{l}\text { Spaansch } \\
\text { Water. }\end{array}$ & $\begin{array}{c}\text { Spaansche } \\
\text { Haven. }\end{array}$ & $\begin{array}{c}\text { Boca } \\
\text { labadera. }\end{array}$ & Westpunt. \\
\hline Actiniaria. & & & & & \\
\hline Edwardsia horstii Pax ... . . . . . . . & - & + & - & - & - \\
\hline Isoedwardsia curaçaoensis $\mathrm{Pax}$. . . . . . . . & - & + & - & - & - \\
\hline Condylactis passiflora Duch. \& Mich. . . . . . & + & + & - & - & - \\
\hline Anemonia antillensis $\mathrm{Pax} \quad . \quad . . . . . .$. & - & - & - & - & + \\
\hline Actinia bermudensis (Mc Murr.) . . . . . . . . & $t$ & - & - & - & - \\
\hline Bunodeopsis globulifera Verr. . . . . . . . . & - & + & + & - & $\therefore$ \\
\hline Bunodactis curaçaoensis Pax . . . . . . . . . . & - & - & - & + & - \\
\hline Bunodactis stelloides (Mc Murr.) $\cdot$. . . . . . . . & -- & - & + & - & - \\
\hline Bunodosoma granuliferum (Les.) . . . . . . . .. & - & - & - & + & - \\
\hline Bunodosoma kükenthali $\mathrm{Pax}$. . . . . . . . . . & + & - & - & + & - . \\
\hline Asteractis conchilega (Duch. \& Mich.) . . . . . & - & + & + & - & - \\
\hline Phellia rufa Verr. . . . . . . . . . . . . & + & - & - & - & - \\
\hline Phellia pseudo-roseni Pax . . . . . . . . . . . & + & - & - & - & - \\
\hline Calliactis tricolor (Les.).$\ldots \ldots$ & + & - & - & - & - \\
\hline Aiptasia tagetes (Duch. \& Mich.) . . . . . . . & - & + & - & - & - \\
\hline Bartholomea annulata (Les.) . . . . . . . . . . & + & + & - & - & - \\
\hline Lebrunia danae (Duch. \& Mich.) . . . . . . . . & - & + & - & - & - \\
\hline Phymanthus crucifer (Les.) . . . . . . . . . & + & + & - & - & - \\
\hline Stoichactis helianthus (Ell.) . . . . . . . . . . . & + & - & - & - & - \\
\hline Zoantharia. & & & & & \\
\hline Zoanthus sociatus (Ell.) . . . . . . . . . & + & + & - & - & - \\
\hline Zoanthus pulchellus (Duch. \& Mich.). . . . . . & + & - & - & - & - \\
\hline Palythoa caribaeorum. Duch. \& Mich.. . . . . . & + & - & - & - & - \\
\hline 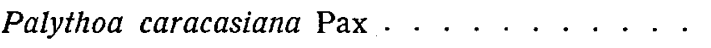 & + & - & - & - & - \\
\hline 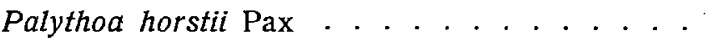 & - & - & - & - & + \\
\hline Isaurus duchassaingi var. microtuberculata Pax. & + & - & - & - & - \\
\hline Isaurus gelatinosus $\mathrm{Pax} . . . . . . . .$. & + & - & - & - & - \\
\hline Ceriantharia. & & & & & \\
\hline Ceriantheopsis spec. . . . . . . . . . . . . . . & + & - & + & - & - \\
\hline
\end{tabular}

In der Caracas-Bai sammelte VAN DER HORST 15, im Spaansch Water 10 Arten, aber nur 4 Spezies sind beiden Fundorten gemeinsam. Über die anderen Sammelplätze (Spaansche Haven, Boca labadera, Westpunt) lässt sich kaum etwas aussagen, da dort nur wenige Stücke konserviert wurden.

\section{Actiniaria.}

Edwardsia horstii nov. spec.

(Taf. IX, Fig. 11).

Fundorte: Curaçao, Spaansch Water, 4. Mai 1920 (40 Exemplare). Curaçao, Spaansch Water, 5. Mai 1920 (3 Exemplare).

Der Körper der meisten Exemplare ist lang gestreckt und etwa zylindrisch; bei einigen ist eine Verschmälerung des distalen Teiles des Scapus sowie des Capitulum deutlich erkennbar. Die Physa ist sehr schwach entwickelt und kann in den Scapus vollständig eingezogen werden. Bei fast allen Exemplaren sind an der Physa kleine Steinchen angeheftet. Nemathybome fehlen der Physa vollständig. Der Scapus, der in konserviertem Zustande eine schmutzig-braune Farbe aufweist, zeigt ein runzeliges Aussehen, das nicht nur durch eine Kontraktion in der Längsrichtung, sondern vor allem durch den Besitz von Nemathybomen bedingt ist. Die Nesselhöcker treten besonders an den Stellen des Scapus 
auf, die sich bei manchen Individuen schon äusserlich in Gestalt winziger grauer Flecken von dem Braun der Umgebung abheben. Ihre Verteilung ist durchaus unregelmässig. Jedenfalls zeigen sie nirgends die Tendenz, sich in Längsreihen anzuordnen. Die Mesoglöa des Scapus enthält ziemlich viele Bindegewebszellen, übertrifft an Dicke die beiden primären Keimblätter zusammen und ist an ihrer ektodermalen Seite in zahlreiche Zapfen und Papillen ausgezogen (Fig. 2). Das Entoderm des Scapus ist sehr zart-und-wird aussen von einer. kräftigen Cuticula überzogen, die etwa doppelt so dick ist wie das Ektoderm. Bei den meisten Exemplaren ist die Cuticula mit feinen Schlammpartikeln inkrustiert. Acht sehr deutliche, auch auf der Photographie (Taf. IX, Fig. 11) erkennbare Längsfurchen des Scapus entsprechen der Insertion der Mesenterien. Das Capitulum, das wie die Tentakel in konserviertem

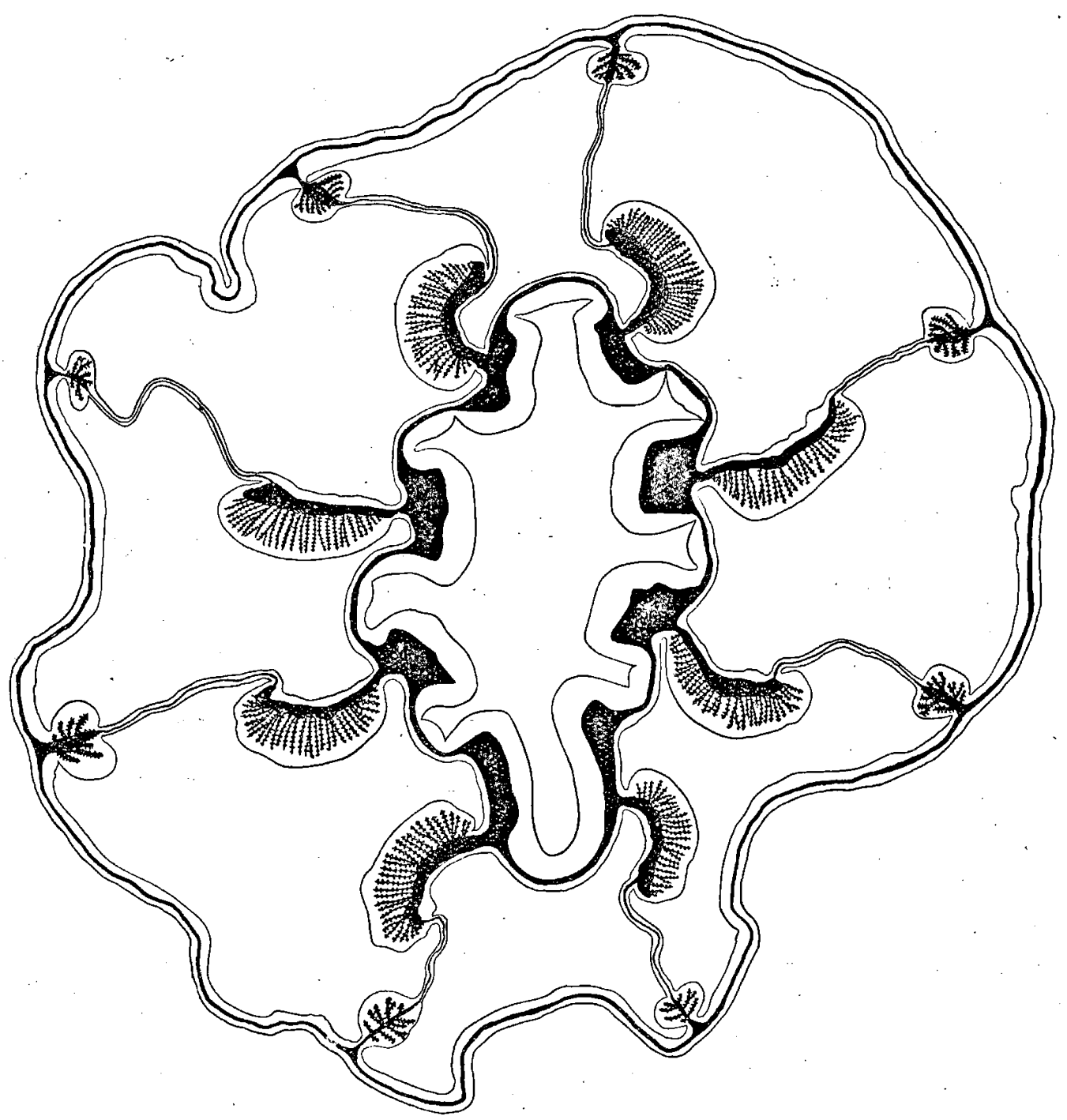

Fig. 1. Querschnitt durch Edwardsia horstii in Schlundrohrhöhe.

Zustande gelblich-weiss gefärbt und durchscheinend ist, erreicht nur ein Sechstel der Länge des Scapus. Die Längsfurchen des Scapus setzen sich auf dem Capitulum als zarte, mehr oder minder undeutliche Längslinien fort. Das Ektoderm des Capitulum ist von sehr zarter Beschaffenheit, weist aber die gleiche Dicke wie die glatte, nirgends Zapfenbildungen zeigende Mesoglöa auf (Fig. 1). Eine Cuticula fehlt im Bereiche des Capitulum.

Unter dem reichlich vorhandenen Material befindet sich ein Exemplar, das infolge vorheriger Betäubung mit Chloralhydrat in völlig ausgestrecktem Zustande fixiert werden konnte (Taf. IX, Fig. 11). Die im Folgenden angegebenen Masse wurden an diesem Stück genommen, das gleichzeitig zur Herstellung der in Figur 1 und 2 dargestellten Schnitte diente: Die Gesamtlänge des Körpers beträgt $21 \mathrm{~mm}$, wovon $18 \mathrm{~mm}$ auf den Scapus einschliesslich der völlig eingezogenen Physa und $3 \mathrm{~mm}$ auf das 
Capitulum entfallen. An der breitesten Stelle misst der Scapus $15 \mathrm{~mm}$, die Länge der Tentakel beträgt $5 \mathrm{~mm}$ : Die übrigen weniger gut fixierten Exemplare erreichen zum Teil nur die Hälfte dieser Masse.

Das Peristom ist konkav und mit 8 feinen radial verlaufenden Linien bedeckt, die kaum stärker als diejenigen des Capitulum hervortreten. Die von mir untersuchten Exemplare wiesen 16 kegelförmige, isakmäische,-glatte Tentakel auf, die keine Andeutung eines äusserlich erkennbaren Reliefs zeigten. Die Mundöffnung ist kreisrund. Gonidialtuberkel fehlen. Das. im Querschnitt ovale Schlundrohr ist $6 \mathrm{~mm}$ lang, erreicht also etwa ein Drittel der Körperlänge und ist somit länger als das Capitulum. Acht symmetrisch gelegene Längsfurchen des Schlundrohrs, von denen eine als Siphonoglyphe entwickelt ist, werden durch acht polsterförmige Vorsprünge der Mesoglöa voneinander getrennt. Während die Mesoglöa des Schlundrohrs lokale Verdickungen aufweist, zeichnet sich das hohe Schlundrohr-



Fig. 2. Querschnitt durch Edwardsia horstii unterhalb des Schlundrohrs.

ektoderm durch eine sehr gleichmässige Dicke aus. Acht vollständige, nach dem Edwardsia-Typus angeordnete Mesenterien verbinden das Schlundrohr mit der Körperwand. Unvollständige Mesenterien fehlen und sind auch nicht durch mesoglöale Auswüchse der Körperwand angedeutet. Die Retraktoren sind sehr kräftig entwickelt und ausgesprochen circumscript. In der Schlundrohrregion liegen sie nicht in der Mitte zwischen Körperwand und Schlundrohr, sondern schmiegen sich dem Schlundrohr dicht an (Fig. 1). Unterhalb des Schlundrohrs erscheinen sie auf dem Querschnitt als nieren- oder halbmondförmige Gebilde (Fig. 2), deren kräftige Entwicklung in einem auffälligen Gegensatze zu der geringen Dicke der Mesenterien steht. Die Mesenterialfilamente sind mit Flimmerstreifen ausgestattet. Die Parietalmuskeln treten uns auf dem Querschnitt als zierlich verzweigte Bäumchen mit jederseits durchschnittlich fünf im spitzen Winkel vom Hauptstamm abgehenden Seitenzweigen entgegen, die ihrerseits wie- 
derum Äste höherer Ordnung entsenden. Die Gonadenregion ist auffallend kurz; alle Mesenterien sind fertil. Das von mir anatomisch untersuchte Exemplar weist reichlich entwickelte Hoden auf. Ein Sphinkter fehlt.

\section{Isoedwardsia curaçaoensis nov. spec.}

Fundort: Cu raça o, Spaansch Water, 20. Mai 1920 (1 Exemplar).

Die Farbe des konservierten Tieres ist grau. Der langgestreckte und annähernd zylindrische Körper gliedert sich in zwei Abschnitte, Scapus und Capitulum. Eine Physa fehlt, wie ich auf Längsschnitten - nachweisen konnte, vollständig. Das aborale Körperende ist abgerundet und mit einer deutlichen Cuticula und Nemathybomen ausgestattet, stimmt in seinem histologischen Bau also mit dem Scapus überein, der ein höckeriges Aussehen besitzt und nur in seiner oberen Hälfte 8 Längsfurchen erkennen lässt. Figur 3 zeigt einen Querschnitt durch einen Teil des Scapus. Zwischen den Ansatz-



Fig. 3. Querschnitt durch einen Teil des Scapus von Isoedwardsia curafaoensis mit den in die Mesoglöa eingebetteten Nemathybomen.

stellen zweier an ihren Parietalmuskeln leicht kenntlichen Mesenterien sehen wir eine Reihe von Hohlräumen, die von Ektoderm und Cuticula überzogen sind. Diese Kavitäten sind lediglich durch Einfaltung der Körperwand entstanden. Daneben zeigt die Abbildung noch fünf quer durchschnittene Nemathybome, die in die Mesoglöa eingebettet sind, wahrscheinlich aber einen auf dem Querschnitt nicht getroffenen feinen Ausführgang besitzen. Eine Anordnung der Nemathybome in Längsreihen ist nicht erkennbar. Vielmehr scheinen die in grosser Zahl vorhandenen Nesselhöcker regellos nebeneinander zu liegen. Die Mesoglöa des Scapus, die vielfach in zierlich verzweigte Zapfen ausgezogen ist, erreicht im proximalen Teile eine grössere Dicke als in der distalen dem Capitulum benachbarten Region. Das zarte Capitulum entbehrt einer Cuticula; Längsstreifen, die den Ansatzstellen der Mesenterien entsprechen, sind nur mit bewaffnetem Auge erkennbar. Das Capitulum ist auffallend kurz. Von der gesamten Körperlänge, die $45 \mathrm{~mm}$ beträgt, entfallen $42 \mathrm{~mm}$ auf den Scapus, $3 \mathrm{~mm}$ auf das Capitulum. Die 16 fadenförmigen Tentakel sind $11 \mathrm{~mm}$ lang, isakmäisch und am freien Ende zugespitzt. Die Gestalt der Mundöffnung ist an dem vorliegenden Exemplar nicht zu erkennen, da das Schlundrohr vollständig ausgestülpt ist. Gonidialtuberkel fehlen. Das Schlundrohr ist merklich länger als das Capitulum; seine Länge darf man auf fast ein Drittel der Körperlänge schätzen. Dịe Zahl der nach dem 
Edwardsia-Typus angeordneten vollständigen Mesenterien beträgt 8; unvollständige Mesenterien fehlen. Die Retraktoren (Fig. 4) sind sehr kräftig und circumscript. Sie unterscheiden sich aber von den

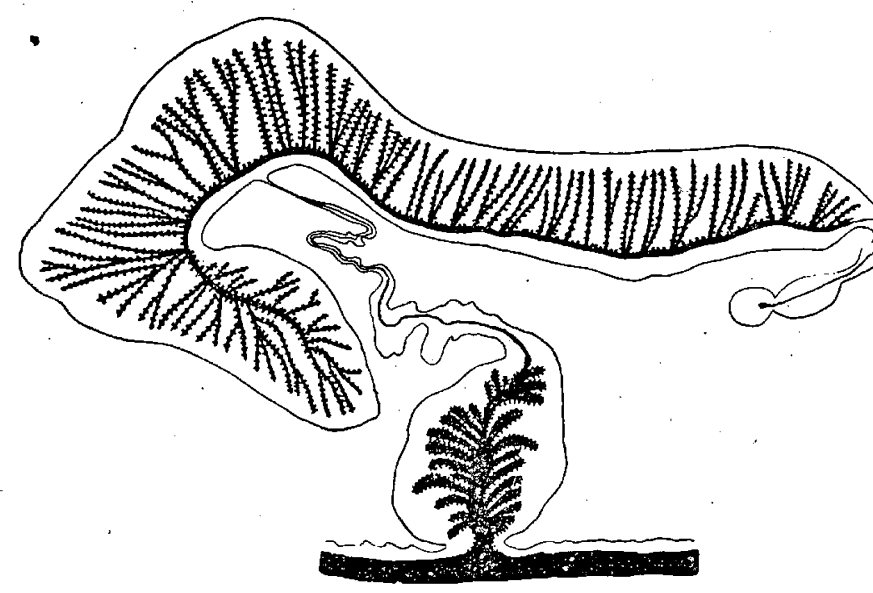

Fig. 4. Retraktor und Parietalmuskel von Isoed wardsia curaçaoensis dicht unterhalb des Schlundrohrs. Längsmuskeln der Mesenterien von Edwardsia horstii dadurch, dass die Bindegewebslamellen fast sämtlich von gleicher Länge, palisadenförmig angeordnet und wenig verzweigt sind. Auch die Zahl der Lamellen ist nicht sehr gross. Wenn also auch der Retraktor als circumscript bezeichnet werden muss, erscheint sein Querschnitt doch nicht sehr hoch, aber verhältnismässig lang. Diese Befunde gelten für die Schlundrohrregion und den dicht unterhalb des Schlundrohrs gelegenen Körperabschnitt. Im proximalen . Teile des Scapus erscheint der Retraktor auf Querschnitten (Fig. 5) als ein kleines, aber dickes Polster. Die Mesenterialfilamente sind mit Flimmerstreifen ausgestattet. Die Parietalmuskeln sind kräftig entwickelt. Auf dem Querschnitt (Fig. 4 u. 5) lassen sie jederseits 8-18 recht kompakte Seitenzweige erkennen, die ihrerseits aber wenig oder gar nicht verzweigt sind. Gonaden waren in dem vorliegenden Individuum nicht entwickelt.

Condylactis passiflora Duch. et Mich.

Fundorte: Curaça o, Caracas-Bai, 3. Mai 1920 (1 Exemplar). Curaçao, Caracas-Bai, 10. Mai 1920 (6 Exemplare). Curaça o, Spaansch Water, 5. Mai 1920 (3 Exemplare).

Die prächtig erhaltenen Tiere haben in der Konservierungsflüssigkeit zwar die dunkelrote Farbe des Mauerblattes eingebüsst, aber die olivgrüne Färbung der Tentakel war noch deutlich zu erkennen, als mir das Material zugeschickt wurde. MC MURRICH (1889) glaubt zwischen den von den Bahama-Inseln stammenden Exemplaren und denjenigen der Bermuda-Inseln einen Unterschied feststellen zu können. „In the Bahama specimens”, schreibt Mc MUR$\mathrm{RICH}$, "the mesogloea between the outer edge of the muscle-band and the insertion of the mesentery into the column wall is thin, and the muscle-band gradually thins out externally. In the Bermuda forms, however, the longitudinal muscle begins abruptly, and the mesogloea external to it is thick with short, stout muscle processes; or, as in the directives with the muscle cells, instead of appearing to cover processes, presenting rather the appearence of here and there dipping down slightly into the mesogloea." Ich habe schion früher (1910) betont, dass ich auf Grund meiner eigenen Untersuchungen die Angaben von

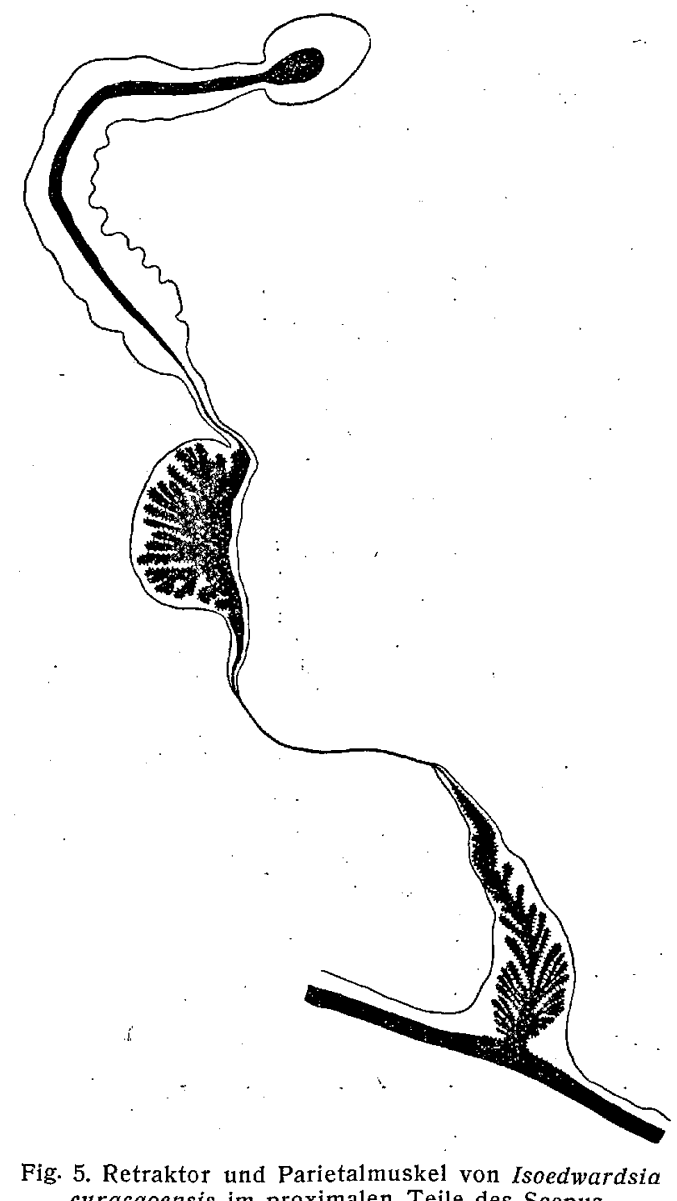
curaçaoensis im proximalen Teile des Scapus.

MC MURRICH nicht bestätigen kann. Nun hat neuerdings WATZL (1922) Exemplare von den BahamaInseln untersucht, die weder dem Bahama- noch dem Bermudatypus Mc MURRICHs völlig entsprachen. Er hält die von Mc MURRICH hervorgehobenen Differenzen „teils für individuelle Variation, teils für ein Ergebnis der Veränderung des Querschnittsbildes bei Kontraktion (Bahamatypus) beziehungsweise Erschlaffung (Bermudatypus)." Nach meinen Erfahrungen sind die Exemplare der Bahama-Inseln im allgemeinen grösser, kräftiger und besitzen schlankere Tentakel mit einer deutlicheren Längsstreifung 
als die Bewohner der Antillen. Das in Curaçao gesammelte Materal stimmt in diesen äusseren Merkmalen durchaus mit der Antillenform überein.

Die Art ist in ihrer Verbreitung auf Westindien und die Bermuda-Inseln beschränkt. In Westindien ist sie nunmehr von Curaçao, Barbados, Thatch Island, St. Thomas, Porto Rico, Jamaica, den Tortugas und den Bahama-Inseln nachgewiesen worden. (Karte 1).

\section{Anemonia antillensis nov. spec.}

Fundort: Cu raça o, Westpunt, 19. Mai 1920 (15 Exemplare).

Eine in konserviertem Zustande bläulich-graue, meist auf Hydroidenstöckchen lebende AnemoniaArt der Sammlung VAN DER HORST stellt nach meinem Dafürhalten eine bisher unbeschriebene Species dar, für die ich den Namen antillensis in Vorschlag bringen möchte. Es läge natürlich nahe, in erster Linie an die von Verrill (1901) von den Bermudas beschriebene Anemonia elegans zu denken, die WATZL (1922) in einer von den Bahama-Inseln stammenden Ausbeute wiedergefunden zu haben glaubt; aber das von VERRILL veröffentlichte Habitusbild stimmt so wenig mit den mir vorliegenden Exemplaren überein, dass ich es nicht wage, die im Litoral von Curaçao gesammelte Anemonia-Art mit A. elegans zu identifizieren:

Anemonia antillensis besitzt eine wohl entwickelte, ausgebreitete, mit kräftigen Basilarmuskeln ausgestattete Fussscheibe, deren Durchmesser etwa $10 \mathrm{~mm}$ beträgt. Bei einigen Exemplaren ist die Fussscheibe nabelförmig eingezogen. Der Limbus hebt sich recht deutlich ab und ist gekerbt. Die Gestalt der ausnahmslos vorzüglich konservierten Tiere ist flach scheibenförmig. Die Höhe des Mauerblattes beträgt $3 \mathrm{~mm}$, doch darf man wohl annehmen, dass dieses Mass am lebenden Tiere den doppelten Betrag erreichen wird, da sämtliche mir vorliegenden Exemplare in der Längsrichtung ein wenig kontrahiert sind. Die Körperwand ist glatt und entbehrt der Saugwarzen und irgend welcher Ausstülpungen oder Anhänge. Den oberen Abschluss des Mauerblattes bildet eine Randfalte, die mit 24 deutlich voneinander abgegrenzten, marginal stehenden Randsäckchen besetzt ist. Eine Fossa ist vorhanden, aber nicht sehr tief. Die Tentakel sind nicht retraktil, randständig, indem sie etwa nur ein Drittel der Mundscheibe einnehmen, von pfriemenförmiger Gestalt und besitzen eine glatte Oberfläche. Ihre Zahl dürfte normalerweise wohl 48 betragen. An einem Exemplar zählte ich 52, an einem anderen 49 Tentakel.

Ein Tentakel zeigte eine Bifurkation, wie sie TUR (1908) früher von Anemonia sulcata beschrieben hat. „Badanie bliższe,” sagt TuR, „nie wykazuje śladów uskodzenia traumatycznego, któreby mogĩo t̃ómaczyć owo rozdwojenie jako objaw wtórny. W innych czulkach tego samego osobnika, oraz u wielu innych osobników tego samego gatunky - nie zauważỹem nic podobnego." Auch an dem vorliegenden Exemplar ist keine Spur einer vorausgegangenen Verletzung des Tentakels zu erkennen.

Die inneren Tentakel von Anemonia antillensis sind länger als die äusseren. Sie sind $10 \mathrm{~mm}$ lang und erreichen an der Basis eine Breite von 2,5. mm. Die ektodormalen Längsmuskeln der Tentakel sind schwach entwickelt, das Entoderm der Tentakel ist pigmentiert. Das Peristom ist flach und lässt die Insertionsstreifen der Mesenterien durchschimmern. Die Mundöffnung ist kreisrund oder oval und liegt niemals auf einem Conus. Das Schlundrohr ist kurz und trägt 24 Längsfurchen; zwei gut differenzierte Schlundrinnen sind schon bei äusserlicher Betrachtung des Tieres deutlich erkennbar. Die Zahl der Mesenterien beträgt 24 Paar, von denen 12 Paar vollständig sind, darunter 2 Paar den Siphonoglyphen entsprechende Richtungsmesenterien. Die Retraktoren sind zwar

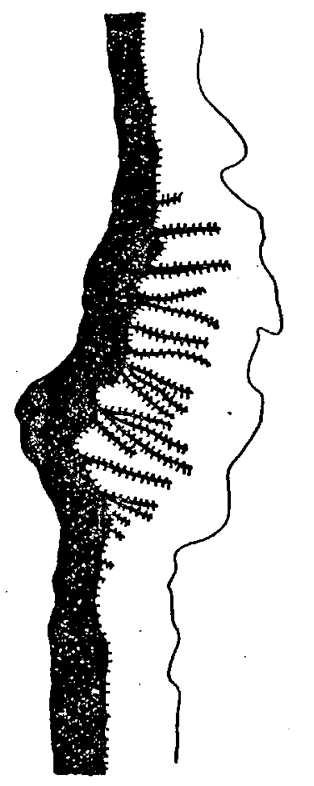

Fig. 6. Querschnit durch den Sphinkter von
Anemonia antillensis. als circumscript $\mathrm{zu}$ bezeichnen, aber doch verhältnismässig schwach entwickelt. Gonaden waren an den von mir untersuchten Tieren nicht entwickelt. Der Sphinkter (Fig. 6) ist circumscript-diffus, besteht aber nur aus wenigen Falten. Zooxanthellen treten im Entoderm in mässiger Zahl auf.

Ich habe mich schon oben in dem Sinne geäussert, dass ich Anemonia antillensis für nicht identisch mit der Anemonia elegans der Bermuda-Inseln .halte. Was nun die von WaTzL (1922) anato- 
misch untersuchte Anemonia elegans der Bahama-Inseln anlangt, so zeigt seine Beschreibung auch erhebliche Unterschiede gegenüber meinen eigenen Befunden. Soweit die unvollständige Beschreibung Watzls einen Vergleich der beiden Arten gestattet, ergeben sich folgende Differenzen:

\section{A. elegans.}

Randsäckchen nicht scharf abgesetzt Länge der Tentakel wechselnd

Innerste Tentakel $3 \mathrm{~mm}$ lang und an der Basis $1 \mathrm{~mm}$ breit

Entoderm der Tentakel nicht pigmentiert Tentakel retraktil?
A. antillensis.

Randsäckchen scharf abgesetzt Innerste Tentakel deutlich am längsten Innerste Tentakel $10 \mathrm{~mm}$ lang und an der Basis $2,5 \mathrm{~mm}$ breit

Entoderm der. Tentakel pigmentiert

Tentakel nicht retraktil

Actinia bermudensis (Mc. Murr.).

Fundort: Curaçao, Caracas-Bai, 13. Mai 1920 (1 Exemplar).

Nach Mc MurRich (1889) hat Actinia bermudensis einen meso-entodormalen Sphinkter. Der Ringmuskel des mir vorliegenden Stückes ist jedoch typisch entodermal und stimmt im wesentlichen mit denjenigen von Actinia equina überein. Vielleicht hat MC MURRICH ein ungünstig geführter Schnitt vorgelegen. Sollte diese Vermutung nicht zutreffen, dann müsste die Spezies bermudensis wohl aus der Gattung Actinia entfernt und für das in Curaçao gesammelte Exemplar eine neue Art aufgestellt werden. A. bermudensis war bisher nur von den Bermuda- und Bahama-Inseln bekannt.

\section{Bunodeopsis globulifera Verr.}

Fundorte: Curaçao, Spaansch Water (Mangrove), 7. April 1920 (2 Exemplare). Curaçao, Spaansche Haven, 10. April 1920 (3 Exemplare). Curaçao, Spaansche Haven, 16. April 1920 (15 Exemplare).

Das mir vorliegende Material stimmt in allen wesentlichen Punkten mit der Beschreibung des äusseren Habitus überein, die VerRILL (1899 u. 1900) auf Grund seiner Beobachtungen am lebenden Tier gegeben hat, sowie mit den durch eine Reihe von Bildern beglaubigten Befunden der anatomischen Untersuchung, die wir DUERDEN (1902) verdanken. Ich darf mich daher mit einigen kurzen Bemerkungen begnügen. Die Farbe des lebenden Tieres nennt VerRILl (1900) „yellowish green, often with dark brown streaks; vesicles yellowish brown”, und DUERDEN (1898) schreibt: „During partial contraction, the colour of the lower part of the column is a bright, opaque yellowish green, with dark brown or black lines or spots. On full inflation, the vesicles and the portion of the column, from which they originate, are a clear, delicate, yellowish brown, due to the yellow cells or zooxanthellae in the endoderm." Als ich die von VAN DER HORST gesammelten, in Formol konservierten Tiere erhielt, waren sie deutlich „zooxanthellengelb” gefärbt; nach ihrer Überführung in Alkohol verschwand die grünlich-gelbe Färbung vollständig. Auch die von DuERDEN (1902) angegebenen Körpermasse stimmen gut mit meinen Beobachtungen überein. Der Durchmesser der Fussscheibe betrug an cien grössten von DuERDEN beobachteten Exemplaren $12 \mathrm{~mm}$, die Körperhöhe schwankte zwischen 5 und $13 \mathrm{~mm}$, und die Länge der inneren Tentakel betrug etwa $10 \mathrm{~mm}$. Ich fand an den konservierten Material folgende Werte: Durchmesser der Fussscheibe12 mm, Höhe der Körperwand 6-7 mm, Länge der inneren Tentakel $8 \mathrm{~mm}$. Im histologischen Bau der Fussscheibe und der Körperwand, einschliesslich der Coelosphaerite, stimmen die mir vorliegenden Exemplare so genau mit DuERDENs (1902) Beschreibung überein; dass ich diese fast wörtlich wiederholen müsste, wollte ich meine Beobachtungen eingehend schildern. Nur in zwei Punkten ergeben sich Verschiedenheiten. Die ektodermale Längsmuskulatur der Körperwand ist viel kräftiger entwickelt (Fig. 7), als dies die Beschreibung und Abbildung DuERDENS erkennen lassen, auch stimmen die von DUERDEN angegebenen Masse der Nesselkapseln nicht ganz mit meinen Beobachtungen überein. Nach DUERDEN sind die ausserordentlich charakteristischen leicht gebogenen dickwandigen Nematocysten, die man in beträchtlicher Zahl im Ektoderm der Cölodendrite antrifft, 38-44 $\mu$ lang, während die grössten von mir in den Nesselorganen der Körperwand beobachteten Kapseln eine Länge von $57 \mu$ erreichten. Das gleiche gilt für die Nesselbewaffnung der Tentakel. 
Nach DUERDEN sollen die dünnwandigen Nematocysten der Tentakel $18-28 \mu$ lang sein; auch hier. habe ich in einer Anzahl von Fällen wesentlich längere, nämlich solche von $34 \mu$ Länge, gefunden. Ich



Fig. 7. Querschnitt durch Bunodegpsis globulifera in Schlundrohrhöhe.

glaube aber nicht, dass diese Unterschiede in den Grössenverhältnissen der Nesselkapseln ausreichen, um die im Litoral von Curaçao heimische Form als eine von der in Jamaica vorkommenden verschiedene Art zu betrachten. Nach VéRRILl (1900) schwankt die Zahi der Tentakel zwischen 18 und 36 . Ich selbst zählte an den von mir untersuchten Exemplaren 36 Tentakel. Eine Vorstellung von der ausserordentlich starken Bewaffnung der Tentakel mit Nesselzellen soll Figur 8 geben, die mit Hilfe des Edingerschen Projektionsapparates ohne irgend welche Schematisierung oder Retouche gezeichnet worden ist. Wir sehen einen Teil eines Längsschnittes, und zwar links das mit dünnwandigen Nematocysten beladene Ektoderm, in dem zufällig gar keine dickwandigen Nesselkapseln getroffen sind, ferner die kräftig entwickelte ektodermale Längsmuskelschicht, die in der Abbildung tief schwarze Mesoglöa mit ihren Ansatzstellen für die entodermale Ringmuskulatur und schliesslich das mit Zooxanthellen besetzte Entoderm.

Das Vorkommen eines circumoralen Sphinkters, das schon DUERDEN (1902) angibt, kann ich bestätigen. Der Sphinkter ist sehr schwach, aber vielleicht doch noch ein ganz klein wenig stärker als bei den Exemplaren von Jamaica; die DUERDEN seiner Beschreibung zu grunde gelegt hat. Nach DuERDEN erreicht das Schlundrohr etwa die Hälfte der Körperlänge. Bei den Exemplaren von Curaçao scheint es nach den von mir angefertigten Längsschnitten etwa drei Viertel der Körperlänge aufzuweisen, doch beruht

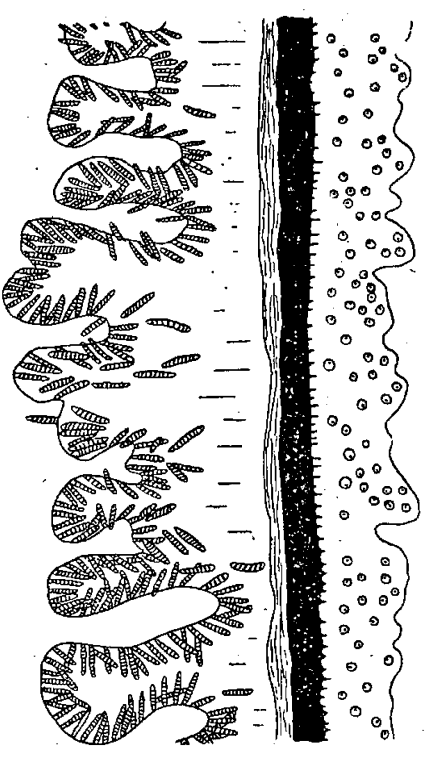

Fig. 8. Teil eines Längsschnittes durch einen Tentakel von
Bunodeopsis globulifera.

diese Angabe lediglich auf einer Schätzung, da genaue Messungen wegen der Kontraktion und der durch sie bedingten Faltenbildungen der Körperwand und des Schlundrohrs nicht möglich sind. VerRILl (1900) 
erwähnt das Vorkommen von „two feeble siphonoglyphs”. Wie DUERDEN (1902) an seinem Material von Jamaica kann ich auch an den in Curaçao gesammelten Tieren selbst bei sorgfältigster histologischer Untersuchung des Schlundrohrs keine Spur einer derartigen Differenzierung wahrnehmen.

Die Anordnung der Mesenterien zeigt die schon von DUERDEN geschilderten Unregelmässigkeiten. Eines der von mir in Querschnitte zerlegten Exemplare wies 18 Paar Mesenterien, darunter 1 Paar Richtungsmesenterien, auf. 9 Paar Mesenterien waren vollständig, die übrigen unvollständig (Fig. 7). Bei einem zweiten Individuum fand ich 26 Paar Mesenterien, wobei allerdings einzelne Paare nur als kleine Bindegewebszapfen entwickelt waren, denen nicht einmal eine Ausbuchtung des Entoderms entsprach. Schon DuERDEN hat die Vermutung ausgesprochen, dass die unregelmässige Entwicklung der Mesenterien mit dem Vorkommen einer unter dem Namen Laceration bekannten Form der ungeschlechtlichen Fortpflanzung zusammenhänge, bei der sich Teile der Fussscheibe loslösen und den Ausgangspunkt für die Entwicklung eines neuen Individuums bilden. Tatsächlich fanden sich unter den am 16. April 1920 im "Spaansche Haven” gesammelten Exemplaren zwei Fussscheibenfragmente, die vermutlich durch Laceration entstanden sind. Um über die Entwicklung der Mesenterien Klarheit zu gewinnen, zerlegte ich die beiden Lacerationsstücke in Querschnitte; leider verdarben die Schnitte aber, so dass diese Frage offen bleiben muss. Eines, der von mir untersuchten Exemplare war steril; ein zweites war zwar fertil, wies aber nur auf zwei Mesenterien Anlagen von Ovarien auf.

Bunodeopsis globulifera gehört zu den seltensten Aktinienarten Westindiens. Sie wurde bisher nur an den Bermuda-Inseln und im Litoral von Jamaica gefunden.

Bunodactis curaçaoensis nov. spec.

Fundort: Curaçao, Boca labadera, 12. Mai 1920 (1 Exemplar).

Fussscheibe und Körperwand sind fleischfarben, die Saugwarzen des Mauerblattes dunkelgrün, die Tentakel grünlich-gelb.

Der Durchmesser der Fussscheibe beträgt $9 \mathrm{~mm}$, die Höhe der Körperwand $5 \mathrm{~mm}$, der Durchmesser der Mundscheibe $11 \mathrm{~mm}$, die Tentakel sind $4 \mathrm{~mm}$ lang bei einem basalen Durchmesser von $11 \mathrm{~mm}$.

Die Fussscheibe ist kräftig entwickelt, ausgebreitet, von annähernd kreisrundem Umriss und mit einem deutlichen Limbus ausgestattet. Die Körperform ist kelchförmig, d. h. das zylindrische Mauer-

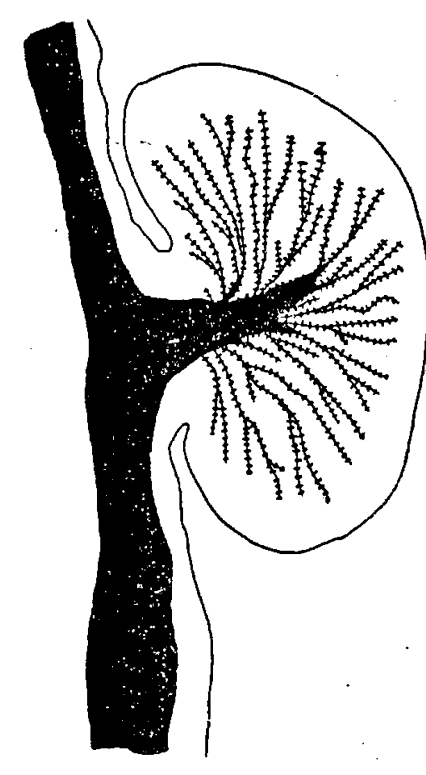

Fig. 9. Querschnitt durch den Sphinkter von Bunodactis curafaoensis. blatt legt oben etwas aus. Die Saugwarzen, die nur das proximale Viertel des Körpers frei lassen, sind in 48 Längsreihen angeordnet und mit Tangstücken, Muschelfragmenten und kleinen Kalkkörnern bedeckt. Auf diese Weise kommt eine Maskierung des Körpers zu stande, die allerdings insofern sehr vergänglich ist, als sie schon beim blossen Anfassen des konservierten Tieres abfällt. Die obersten Saugwarzen stehen marginal. Margo und Fossa sind gut entwickelt, Acrorhagi fehlen. Die Tentakel, die knapp die Hälfte der Mundscheibe einnehmen, sind anscheinend sämtlich von gleicher Länge, mit feinen Längsstreifen und einer abgestumpften Spitze versehen. Das Peristom wird von dem ausgestülpten Schlundrohr bedeckt, das zwei nicht sehr deutlich differenzierte Siphonoglyphen besitzt. Wahrscheinlich sind 24 Paar Mesenterien vorhanden, darunter 2 Paar Richtungsmesenterien. 12 Paar Mesenterien scheinen vollständig zu sein. Bezüglich der Mesenterienverhältnisse kann ich leider keine ganz sicheren Angaben machen, da die von mir angefertigten Schnitte infolge falscher Orientierung im Paraffinblock nicht gut gelungen waren und die Anfertigung neuer Schnitte die Zerstörung eines grossen Teiles des einzigen vorhandenen Exemplars bedingt hätte. Die Retraktoren sind zwar circumscript, aber doch nicht so kräftig, dass

ihr Querschnittsbild sich dem nierenförmigen Typus näherte.

Das mir vorliegende Stück ist weiblichen Geschlechts. Die Ovarien treten auf allen Mesenterien einschliesslich der Richtungsmesenterien auf. Der Sphinkter (Fig. 9) ist circumscript. Die Bindegewebslamellen, die seinen Muskelfasern als Ansatzstellen dienen, sind auffallend zart und dünn. Im Entoderm treten massenhaft Zooxanthellen auf. 


\section{Bunodactis stelloides (Mc Murr.).}

Fundort: Curaçao, Spaansche Haven, 6. Mai 1920 (72 Exemplare).

Bunodactis stelloides scheint innerhalb ihres Verbreitungsareals überall dieselben Standorte zu bevorzugen. "Not unfrequently on the under surface of blocks of coral rock along the shore in shallow water" nennt sie MC MURRICH (1889) in seiner Bearbeitung der Actiniarien der Bahama-Inseln, und VERRILL (1907) schreibt auf Grund seiner Beobachtungen auf den Bermuda-Inseln: „This small species is common, both upon the reefs and ledges, but prefers sheltered spots or the under surfaces of large stones and dead corals, occurring in such places even above low tide." Auch VAN DER HORST hat die Art in Curaçao, wie der Fundortszettel angibt, unter Steinen gesammelt. Die Saugwarzen der Körperwand sind an allen erwachsenen Stücken sehr deutlich entwickelt, an einigen jungen Individuen aber selbst unter der Lupe schwer zu erkennen. Bunodactis stelloides ist vivipar. DuERDEN (1898) beobachtete das Ausstossen der Jungen in Jamaica in den Monaten Juli und August.

Sicher nachgewiesen ist das Vorkommen der "small stellate anemone”, wie VERRILL (1907) die Art nennt, in Jamaica, den Bahama-Inseln und den Bermudas.

\section{Bunodosoma granuliferum (Les.).}

Fundort: Curaçao, Boca labadera, 12. Mai 1920 (3 Exemplare).

Die Art ist in den Antillen (Curaçao, Barbados, Martinique, Guadeloupe, St. Thomas, Loango, Porto Rico, Jamaica) und den Bahama-Inseln verbreitet, fehlt jedoch den Bermuda-Inseln.

\section{Bunodosoma kükenthali Pax.}

Fundorte: Curaçao, Caracas-Bai, 3. Mai 1920 (3 Exemplare). Curaçao, Boca labadera, 12. Mai 1920 (7 Exemplare).

Die Species ist in ihrer Verbreitung auf die Antillen (Curaçao, Barbados, Loango) beschränkt.

\section{Asteractis conchilega (Duch. \& Mich.).}

\section{(Taf. IX. Fig. 13).}

Fundorte: Curaçao, Spaansch Water, 3 April 1920 (1 Exemplar). Curaça o, Spaansche Haven, 11. Mai 1920 (1 Exemplar juv.). Curaçao, Spaansch Water, 15. Mai 1920 (5 Exemplare).

In seinen „Actiniarien der Bahama-Inseln” (1922) hat WATZL darauf aufmerksam gemacht, dass die an den Küsten der Bermuda- und Bahama-Inseln heimische Asteractis flosculifera (Les.) kaum von der im Antillenmeer verbreiteten Asteractis conchilega (Duch. \& Mich.) zu trennen sei. Insbesondere weist er darauf hin, dass die Zahl der Blattorgane, die man früher differentialdiagnostisch verwenden zu können meinte, als Artmerkmal nicht in Frage kommen könne. Dass das Vorkommen von Zooxanthellen im Ektoderm der Tentakel von Asteractis conchilega nicht konstant ist, beweisen die Exemplare von Curaçao. Auf zahlreichen Schnitten habe ich nicht eine einzige parasitische Alge im Tentakelektoderm gefunden. Der von mir früher (1910) beschriebene positive Befund an einigen in Jamaica gesammelten Exemplaren muss also als Ausnahme betrachtet werden. „Im übrigen," schreibt WATZL (1922), "scheint nur ein einziges Merkmal für jede der zwei Aktinienformen verschieden und konstant zu sein, nämlich die Anzahl der Septenpaare. Während nämlich beide Asteractis-Formen 48 Tentakel in 4 Cyclen besitzen, sahen DUERDEN und PAx bei $A$. conchilega (expansa) 48 Paar Septen, davon wenigstens 24 Paar oder alle vollständig, wogegen MC MURRICH und ich bei A. flosculifera stets nur 24 Septenpaare, alle vollständig vorfanden." Die Exemplare der Sammlung vAN DER HORST besitzen 24 vollständige Mesenterienpaare, so dass also auch dieses Merkmal sich als inkonstant erweist. Was die Beurteilung des Sphinkters anlangt, so kann ich WATZL nicht beipflichten. Die mir vorliegenden Exemplare von Asteractis conchilega weisen, soweit ich sie anatomisch untersucht habe, einen circumscripten Sphinkter (Fig. 10) auf, der in seinem Bau durchaus mit dem Ringmuskel der früher von mir 
untersuchten Stücke aus Barbados, Jamaica und Haiti übereinstimmt, sich aber meines Erachtens wesentlich von dem circumscript-diffusen Sphinkter von Asteractis flosculifera unterscheidet. Während

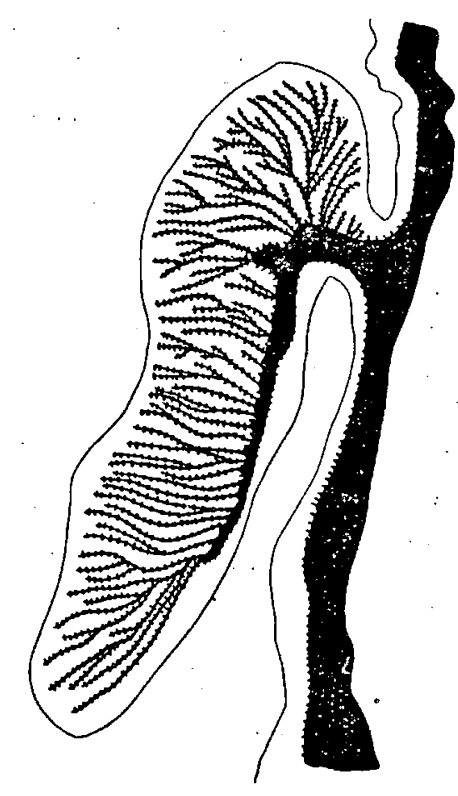

Fig. 10. Querschnitt durch den Sphinkter von Asteractis conchilega. der Sphinkter von $A$. flosculifera sich nach Watzls Abbildungen durch eine beträchtliche Variabilität auszeichnet, scheint mir für den Sphinkter von $A$. conchilega gerade eine bemerkenswerte Konstanz der Form bezeichnend zu sein. Halten wir an der Trennung der beiden Arten fest, so wird das Areal von Asteractis conchilega durch folgende Standorte umschrieben: Curaçao, Barbados, Porto Rico, Haiti, Jamaica, Cuba.

Die Jugendformen von Asteractis conchilega besitzen einen sehr schmalen Randkragen, aber schon kräftig entwickelte Saugwarzen. Taf. IX, Fig. 13 stellt ein jugendliches Exemplar, von oben gesehen, dar.

\section{Phellia rufa Verr.}

(Taf. IX, Fig. 8).

Fundort: Cu raça o, Caracas-Bai, unter Steinen, 1. Mai 1920 (4 Exemplare).

In der Sammlung VAN DER HORST befinden sich 4 Exemplare einer Phellia-Art, die ich ohne Bedenken als Phellia rufa Verr. bestimme, obwohl von dieser Spezies bisher nur Beschreibungen des Äusseren, aber keine Angaben über den anatomischen Bau vorliegen '). Ich benütze gleichzeitig die Gelegenheit, diese Lücke auszufüllen.

Die Farbe der konservierten Tiere ist dunkelbraun. Das grösste Exemplar besitzt im leicht kontrahierten Zustande eine Länge von $94 \mathrm{~mm}$. Der Durchmesser des annähernd zylindrischen Körpers beträgt $15 \mathrm{~mm}$. Diese Masse passen gut zu den Befunden Verrills (1901), der die Länge des lebenden Tieres auf $75-100 \mathrm{~mm}$ und den Körperdurchmesser auf $20-36 \mathrm{~mm}$ beziffert. Die Fussscheibe ist kräftig entwickelt, ohne eigentlich ausgebreitet zu sein (Taf. IX, Fig. 8), runzelig, von derber Beschaffenheit und mit einem deutlichen Limbus gegen den papillenfreien Scapus abgesetzt, dessen runzelige von einer kräftigen Cuticula überzogene Oberfläche etwa das Aussehen von zerknittertem Seidenpapier zeigt. Infolge der derben Beschaffenheit des Scapus schimmern die Insertionsstellen der Mesenterien nicht durch. Das Capitulum ist cuticulafrei. „The external cuticle,” schreibt VerRILl (1907), usually ends distally in an abrupt often flaring edge, above which the column is brighter colored and often partially translucent." Diese scharfe Grenze zwischen Scapus und Capitulum ist auch an den mir vorliegenden Stücken wahrnehmbar. Das Ektoderm des Capitulum ist ausserordentlich zart, seine Mesoglöa, wie diejenige des Scapus, in zahlreiche gegen das Ektoderm vorspringende Zapfen ausgezogen. Der distale Körperrand, der die Grenze zwischen Capitulum und Peristom bildet, ist, wie übrigens auch auf den von Verrill $(1900,1901,1907)$ nach lebenden Tieren entworfenen Abbildungen, nicht sehr deutlich entwickelt; eine Fossa fehlt. Die Tentakel sind kurz, nicht zugespitzt, sondern abgerundet, mit Terminalporen versehen, ausgesprochen randständig und vollständig retraktil. Dass die Tentakel von Phellia rufa kurz, nicht zugespitzt und retraktil sind, zeigt auch eine Betrachtung der VerRILLschen (1907) Abbildungen. Die inneren Tentakel scheinen, soweit der Zustand des konservierten Materials ein Urteil gestattet, länger als die äusseren zu sein. Am lebenden Tiere scheint dies viel deutlicher hervorzutreten, denn VERRILL (1901) sagt: "The inner 12 are often erect and decidedly the largest." An dem grössten der mir vorliegenden Exemplare zählte ich 72 Tentakel. Nach VERRILL (1901)

1) Im westindischen Faunengebiet kommen ausser Phellia rufa noch 4 oder 5 weitere Phellia-Arten vor, nämlich die im Folgenden beschriebene Phellia pseudoroseni, Phellia americana Verr., Phellia simplex Verr., Phellia roseni Watzl und, falls die Vermutung von ANDres (1883), dass Capneopsis solidaginis Duch. \& Mich. eine Phellia-Art sei, sich bewahrheiten sollte, Phellia solidaginis (Duch. \& Mich.). In WATZLS „Actiniarien der Bahama-Inseln” (1922) findet sich die Bemerkung: „Schliesslich führt Pax (Westind. Aktinien 1910, p. 288) noch eine „Phellia simplex" vor den Bermudas an, deren Entdeckung und Beschreibung mir unbekannt geblieben ist". Dieser Satz könnte in dem Leser leicht den Verdacht erwecken, dass mir eine grobe Namenverwechslung untergelaufen ist. Ich möchte daher darauf hinweisen, dass VERRILL die Beschreibung von Phellia simplex schon 1901 in seinen "Additions to the Fauna of the Bermudas from the Yale Expedition of 1901" (Transact. Connecticut Acad. Vol. 11, p. 48) veröffentlicht und die Art 1907 in seinem grossen Werk „The Bermuda Islands” (Transact. Connecticut Acad. Vol. 12, p. 279) nochmals erwähnt"hat. 
kann ihre Zahl bei den grössten Individuen auf 96 bis 120 steigen. Die Längsmuskulatur der Tentakel ist im wesentlichen ektodermal und stellenweise rudimentär. Das Schlundrohr erreicht mehr als die Hälfte der Körperlänge, ist von zarter Beschaffenheit und mit zwei sehr deutlichen, auch histologisch gut differenzierten Schlundrinnen versehen. Auf Querschnitten erkennt man, dass das Schlundrohr im ganzen 30 Längsfurchen aufweist. Schlundrohrzipfel fehlen. Der Durchmesser der Schlundrohrwand beträgt $112 \mu$, wovon $51 \mu$ auf das Ektoderm, $49 \mu$ auf die Mesoglöa und nur $12 \mu$ auf das Entoderm entfallen. Ektodermale Längsmuskeln fehlen dem Schlundrohr. Die Zahl der Mesenterien schwankt nach meinen Beobachtungen. zwischen 35 und 36 Paaren. Diese Zahlen kommen dádurch zu stande, dass die Paare des vierten Zyklus unregelmässig entwickelt sind. Nur 6 Mesenterienpaare sind vollständig (darunter 2 Paar Richtungsmesenterien) und als typische Makroknemen im Sinne von STEPHENSon (1920) mit stark circumscripten Retraktoren entwickelt. Ihr Querschnitt erinnert an die Abbildung, die STEPHENSON (1920) von Phellia phassonesiotes gibt. Alle übrigen Mesenterien sind Mikroknemen mit rudimentären Retraktoren, aber leidlich entwickelten Parietobasilarmuskeln. Akontien sind reichlich vorhanden; Cincliden fehlen sicher. Das von mir anatomisch untersuchte Exemplar war steril.

Der Sphinkter ist kräftig entwickelt, mesoglöal und von beträchtlicher Länge. Seine Muskelmaschen sind fast überall quer gestreckt und füllen nirgends die ganze Breite der Mesoglöa aus. Allerdings werden die Maschen in dem kolbenförmigen distalen Teile des Sphinkters nur durch einen sehr schmalen, auf beiden Seiten gleich breiten Streifen muskelfreien Bindegewebes von den primären Keimblättern getrennt. In dem langen, stielförmigen proximalen Teile des Sphinkters beträgt die Entfernung vom Ektoderm etwa das Doppelte der Sphinkterbreite, während der Abstand vom Entoderm etwa der Breite des Sphinkters gleichkommt. Auf einer sehr langen Strecke läuft hier der Sphinkter in stets gleicher Breite und im gleichen Abstande vom Entoderm dahin. Im distalen Teile des Sphinkters sind die Maschen gröber und unregelmässiger angeordnet als im proximalen Teile; wo sie horstweise zusammenliegen und eine. Art vertikaler Schichtung erkennen lassen. Bemerkenswert erscheint schliesslich der Umstand, dass parallel zu dem mesoglöalen Sphinkter eine kräftig entwickelte entodermale Ringmuskelschicht verläuft.

Phellia rufa ist bisher mit Sicherheit nur von den Bermuda-Inseln nachgewiesen worden. Wahrscheinlich ist sie auch an der Küste von Jamaica heimisch, da Duerdens (1898) „Phellia clavata (Duch. \& Mich.)" wohl nichts anderes als Phellia rufa Verr. sein dürfte.

Phellia pseudoroseni nov. spec.

(Taf. IX, Fig. 7).

Fundort: Curaça o, Caracas-Bai, 18. Mai 1920 (2 Exemplare).

Die Farbe der konservierten Tiere ist gelblich-grau. Ihre Körperform ist zylindrisch. Der Körperdurchmesser beträgt 5-7 mm, die Körperhöhe 22-26 mm. Die Fussscheibe misst im Durchmeśser 5-6 $\mathrm{mm}$, die Mundscheibe 8-9 mm. Die Länge der Tentakel beträgt $3 \mathrm{~mm}$.

Die Fussscheibe ist bei beiden mir vorliegenden Exemplaren nicht ausgebreitet, sondern nabelförmig eingezogen. Ein Limbus fehlt. Die Gliederung der Körperwand in Scapus und Capitulum ist äusserlich nicht so deutlich wahrnehmbar wie bei Phellia rufa Verr. Der Scapus ist von sehr derber Beschaffenheit, papillenfrei, aber in seiner ganzen Ausdehnung dicht mit feinen Runzeln bedeckt. Die borkenartige Cuticula, die dem Scapus ein so charakteristisches Aussehen verleiht, fehlt dem Capitulum. Margo und Fossa sind nicht vorhanden. Die Tentakel, deren Zahl etwa 70 beträgt, sind kurz, kegelförmig, ausgesprochen randständig und stehen sehr dicht nebeneinander. Die Mundöffnung ist spaltförmig und $2 \mathrm{~mm}$ lang. Das Schlundrohr ist mit zwei wohl differenzierten und symmetrisch gelegenen Schlundrinnen ausgestattet. Daneben weist es noch 12 weitere Längsfurchen auf, die durch nicht sehr hohe, polsterförmige Verdickungen der Mesoglöa von einander getrennt werden. 6 Mesenterienpaare, darunter 2 Paar Richtungsmesenterien, sind vollständig und mit sehr. kräftigen, auf dem Querschnitt etwa nierenförmigen Retraktoren versehen. Die Zahl der Mikroknemen vermag ich nicht mit Sicherheit anzugeben, da das von mir zur Untersuchung verwandte Stück der Körperwand auf der Innenseite teilweise mazeriert war. Wahrscheinlich waren 24. Paar Mikroknemen. vorhanden. Die Zahl der Akontien dürfte 
erheblich geringer sein als bei Phellia rufa Verr. Das von mir anatomisch untersuchte Exemplar war steril. Zooxanthellen fehlen.

Der Sphinkter ist kräftig entwickelt, mesoglöal und von erheblicher Länge. Sein verjüngtes distales Ende reicht eine beträchtliche Strecke in das Capitulum hinein. Besonders deutlich zeigt dies Figur 11, auf der ein kleiner schwarzer Pfeil die histologisch sehr scharfe Grenze zwischen Capitulum



Fig. 11. Querschnitt durch die Körperwand von eingebettet liegt. In die Mesogloa (schwa und Scapus bezeichnet. Trotz seiner kräftigen Entwicklung füllt der Sphinkter nirgends die ganze Mesoglöa aus. Dort, wo er in den Scapus übertritt, schwillt er zunächts beträchtlich an, um dann allmählich wieder an Breite abzunehmen und in annähernd gleicher Breite in geringem Abstande von dem Entoderm zu verlaufen (Fig. 11). Das proximale Ende des Sphinkters ist zugespitzt. In der Anordnung der Muskelmaschen ähnelt der Ringmuskel von Phellia pseudoroseni demjenigen von Phellia rufa. In der kräftigen Entwicklung einer auch in der Sphinkterregion vorhandenen entodermalen Ringmuskelschicht stimmen beide Arten gleichfalls überein. Wesentlich grösser ist wohl aber die Ähnlichkeit zwischen Phellia roseni und P. pseudoroseni. Wenn ich mich trotzdem entschlossen habe, die vorliegende Art als eine neue Species $z u$ betrachten, so war für mich ausser der Verschiedenheit der Färbung, dem Fehlen einer Randfalte und der stärkeren Furchung des Schlundrohrs vor allem die abweichende Beschaffenheit des Sphinkters massgebend. WATzL (1922) hebt ausdrücklich hervor, dass der Sphinkter von Phellia roseni im obersten Teile die Mesoglöa ganz erfüllt. Dies trifft für den Sphinkter von Phellia pseudoroseni keinesfalls zu.

Calliactis tricolor (Les.).

Fundort: Curaça o, Caracas-Bai, 26. April 1920 (4 Exemplare).

Diese von DUERDEN (1902) anatomisch untersuchte Art scheint in Westindien weit verbreitet zu sein. Sie ist bisher von St. Vincent, Barbados, St. Thomas, Porto Rico, Jamaica, Cuba und South Carolina nachgewiesen worden. Neu für Curaçao.

\section{Aiptasia tagetes (Duch. \& Mich.).}

(Taf. IX. Fig. 14).

Fundorte: Curaçao, Spaansch Water, 7. April 1920 (6 Exemplare) Curaçao, Spaansch Water, 21. Mai 1920 (15 Exemplare). Curaça o, Spaansch Water, 25. Mai 1920 (36 Exemplare). Curaçao, Spaansch Water, an Mangrovewurzeln, ohne Datum (12 Exemplare).

In meinen „Studien an westindischen Aktinien” (1910) habe ich eine Art, deren Tentakel Nesselleisten besitzen, als Aiptasia tagetes (Duch. \& Mich.) beschrieben. WaTZL (1922) hat mit Recht darauf hingewiesen, dass diese Identifizierung nicht zutreffend sein könne, da die "Bartholomea tagetes" von Duchassaing und MichelotTi glatte Tentakel habe. Diese Tatsache habe ich 1910 übersehen. DuchasSAING und MichELOTTIS Bartholomea tagetes ist also eine Aiptasia, während meine Aiptasia tagetes von Jamaica zur Gattung Bartholumea gestellt werden und einen neuen Namen erhalten muss. Ich schlage vor, sie Bartholomea pseudotagetes zu nennen.

Die. echte Aiptasia tagetes ist in der vorliegenden Sammlung durch 69 zum Teil prächtig konservierte Exemplare vertreten. Wie an der Küste der Bermuda-Inseln erweist sich die -Art auch in Curaçao als ein charakteristischer Bewohner der Mangrove. „The most prolific locality”, schreibt VERRILL (1901), "was the mangrove swamp at Fairy Lands, where it occurred in great numbers, in April, 
attached to the fallen and floating mangrove leaves and twigs". In grossen Kolonien, deren Bildung durch die häufig vorkommende Fortpflanzung durch Laceration wesentlich erleichtert wird, überzieht Aiptasia tagetes die Stelzwurzeln der Mangrovebäume (Taf. IX, Fig. 11). Die Art kommt in Curaçao, St. Thomas, Porto Rico, Jamaica, an den Bahama-Inseln (New Providence, Andros) und den Bermudas vor.

\section{Bartholomea annulata (Les.).}

(Taf. IX, Fig. 6 u. 10).

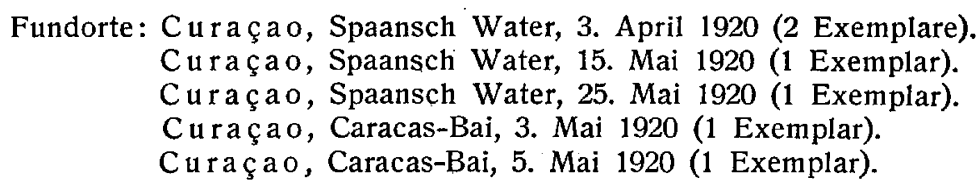

Schon 1910 habe ich innerhalb der Gattung Aiptasia zwei Gruppen unterschieden, die Dactyliophorae mit ringförmig oder spiralig verlaufenden Nesselleisten an den Tentakeln und die Psilonemata mit völlig glatten Tentakein ohne Andeutung von Nesselleisten. STEPHENSON (1920) und WaTzL (1922) sind noch einen Schritt weiter gegangen und haben die beiden in ihrer Nesselbewaffnung so verschiedenen Artengruppen auf zwei verschiedene Gattungen (Aiptasia und Bartholomea) verteilt. Die in der vorliegenden Sammlung durch 5 Exemplare vertretene "Aiptasia" annulata muss nach der von STEPHENSON und WATZL vorgenommenen Einteilung zur Gattung Bartholomea gestellt werden. Bald nach Erscheinen meiner „Studien an westindischen Actinien" (1910) hat HAusDing (1913) Zweifel an der Richtigkeit der Deutung der Nesselleisten der Psilonemata geäussert, indem er darauf hinwies, dass die Tentakel von Metridium dianthus bei Vitalfärbung mit Neutralrot ringförmige Wülste zeigen, die äusserlich stark an die von mir beschriebenen- Nematotänien der BartholomeaArten erinneren. Er hält es also tür möglich, dass die Nematotänien keine morphologisch fixierten Nesselorgane, sondern bei der Kontraktion der Tentakel vorübergehend auftretende wulstförmige Verdickungen seien, und „möchte dazu anregen, die Untersuchung noch einmal von diesem Gesichtspunkte aus vorzunehmen". Selbstverständlich ist die Form der Nematotänien bis zu einem gewissen Grade von dem Kontraktionszustande des Tentakels abhängig. Taf. IX, Fig. 6 zeigt ein erwachsenes Exemplar, bei dem die entodermale Ringmuskulatur der Tentakel so stark kontrahiert ist, dass sämtliche Fangarme ein, rosenkranzförmiges Aussehen annehmen. Taf. IX, Fig. 10 führt uns ein jüngeres Individuum mit geringerer Kontraktion der Tentakel vor. Die Verschiedenheit im äusseren Habitus ist unverkennbar. Aber selbst bei Tieren im $\mathrm{Zu}-$ stande völliger Expansion der Tentakel treten die Nemato-

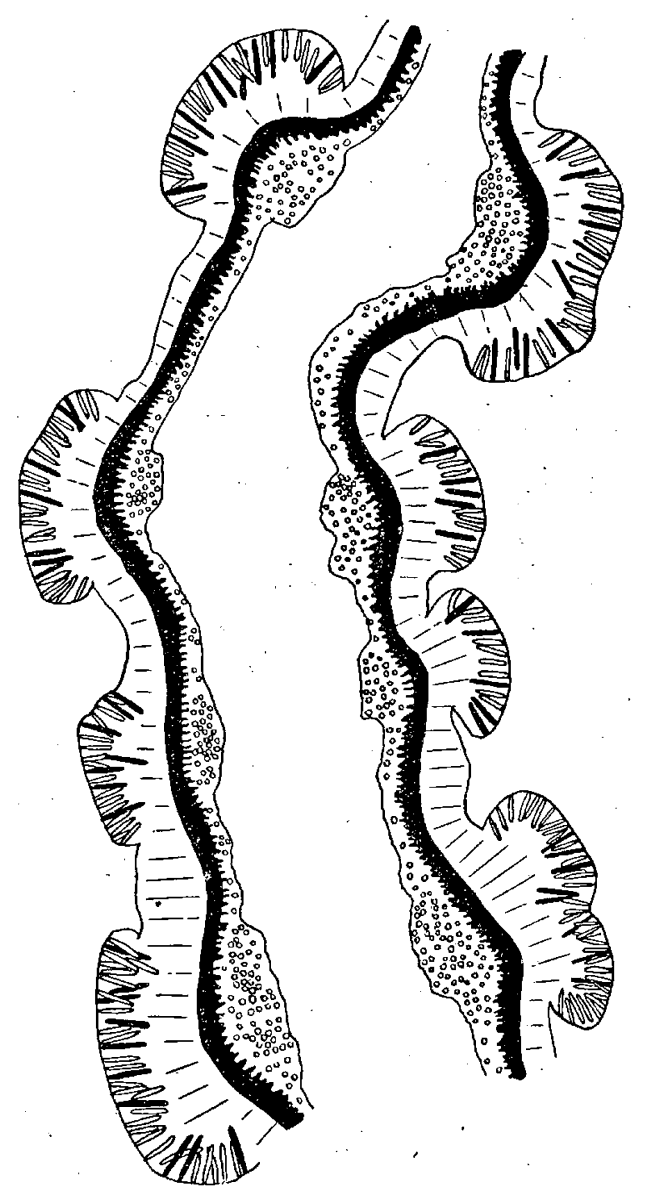

Fig. 12. Teil eines Längsschnittes durch einen Tentakel von Bartholomea annulata tänien als morphologisch wohl abgegrenzte Organe deutlich hervor. Auf Längsschnitten durch einen Tentakel (Fig. 12) erscheinen sie als verdickte Ektodermleisten, in denen es zu einer Anhäufung dünnwandiger und in geringerer Zahl auch dickwandiger Nematocysten kommt.

Bartholomea annulata bewohnt das Litoral der Kleinen (Curaçao, Barbados, Guadeloupe, St. Thomas) und Grossen Antillen (Porto Rico, Jamaica), der Bahama- (New Providence, Hog Island) und Bermuda-Inseln. 
Lebrutia danae (Duch. \& Mich.).

Fundort: Curaça o, Spaansch Water, 26. Mai 1920 (1 Exemplar).

Das einzige Exemplar von Lebrunia danae in der Sammlung VAN DER HORST gehört der schon von DUerden (1898), VerRILL (1907) und PAX (1910) erwähnten olivgrünen Farbenvarietät an. Die Art war bisher von St. Thomas, Jamaica, den Tortugas, Bahama-Inseln und Bermudas bekannt.

\section{Phymanthus crucifer (Les.).}

Fundorte: Curaça o, Caracas-Bai, 23. April 1920 (1 Exemplar). Curaça o, Spaansch Water, 1. Mai 1920 (1 Exemplar):

Das Areal von Phymanthus crucifer umfasst die Antillen (Curaçao, Barbados, St. Thomas, Porto Rico, Jamaica), die Bahama- und die Bermuda-Inseln (Karte 1).

\section{Stoichactis helianthus (Ell.).}

\section{(Taf. IX. Fig. 1).}

Fundorte: Curaça o, Caracas-Bai, 1. Mai 1920 (5 Exemplare). Curaçao, Caracas-Bai, ohne Datum (1 Exemplar).

In meinen "Studien an westindischen Actinien” (1910) hatte ich darauf hingewiesen, dass Exemplare von Stoichactis helianthus, die an verschiedenen Standorten gesammelt worden waren, sehr erhebliche Grössendifferenzen zeigten, während Individuen, die der gleichen Lokalität entstammten, eine gewisse Konstanz der Körpermasse aufwiesen. Gleichzeitig hatte ich die Vermutung ausgesprochen, dass bei dieser Grössendifferenzierung nach Standorten die Verteilung des Salzgehaltes eine Rolle spiele. In Übereinstimmung mit diesen früheren Befunden erwiesen sich sämtliche von mir im Rijksmuseum van Natuurlijke Historie in Leiden untersuchten Stücke, die an der Küste der holländischen Insel Aruba gesammelt worden waren, als ausgesprochen kleinwüchsige Formen (PAX 1924). Dagegen kommen in der Caracas-Bai an der Südküste von Curaçao grosswüchsige und kleinwüchsige Exemplare nebeneinander vor, wie folgende Tabelle zeigt, in der sämtliche Masse in Millimetern angegeben sind:

\begin{tabular}{l|c|c|c|c}
\hline Exemplar & $\begin{array}{c}\text { Durchmesser der } \\
\text { Fussscheibe }\end{array}$ & Körperhöhe & $\begin{array}{c}\text { Durchmesser der } \\
\text { Mundscheibe }\end{array}$ & $\begin{array}{c}\text { Durchmesser der } \\
\text { Mundöffnung }\end{array}$ \\
\hline I & 27 & 10 & 39 & 1 \\
II & 30 & 23 & 41 & 2 \\
III & 29 & 18 & 47 & 2 \\
IV & 30 & 16 & 49 & 21 \\
V & 37 & 11 & 52 & 18 \\
VI & 49 & 18 & 88 & \\
\end{tabular}

Dass es sich bei den kleinwüchsigen Exemplaren nicht etwa um Jugendformen handelt, beweist der Umstand, dass sie wohl entwickelte Gonaden besitzen. Auch die, Vermutung, es lägen vielleicht zwei äusserlich sehr ähnliche Arten vor, ist hinfällig; denn in anatomischer Hinsicht stimmen grosswüchsige und kleinwüchsige Individuen vollkommen überein. Das gilt insbesondere auch für den Bau des Sphinkters.

Nach dem gegenwärtigen Stande unserer Kenntnisse kornmt Stoichactis helianthus an den Kleinen (Aruba, Curaçao, Barbados, Guadeloupe, St. Thomas) und Grossen (Porto Rico, Haiti, Jamaica) Antillen, den Tortugas und den Bahama-Inseln (New Providence, Andros Island) vor. Der Fauna der BermudaInseln fehlt die Art. 
Zoantharia.

Zoanthus sociatus (E11.).

(Taf. IX, Fig. 4 u. 5).

Fundorte: Curaçao, Caracas-Bai, 26. April 1920 (3 Kolonien).

Curaça o, Caracas-Bai, 5. Mai 1920 (1 Kolonie).

Curaça o., Spaansch Water, 15. Mai 1920 (1 Kolonie).

Die Sammlung VA.N DER HORST enthält fünf intensiv braun gefärbte Kolonien dieser bisher von Dominica, Guadeloupe, Porto Rico, Haiti, Jamaica, den Bahama- und den Bermuda-Inseln nachgewiesenen Species. Während drei Kolonien den gewöhnlichen Habitus von Zoanthus sociatus (Taf. IX, Fig. 4) zeigen, weicht eine durch ihren Wuchs nicht unwesentlich von diesem Typus ab (Taf. IX, Fig. 5). Die einzelnen Polypen dieser Kolonie sind gedrungener und durch geringere Zwischenräume von einander getrennt, als es normaler Weise der Fall ist. Auch das Cönenchym zeigt eine kräftigere Entwicklung. Da sich aber im anatomischen Bau keine Verschiedenheiten ergeben, trage ich kein Bedenken, auch diese habituell abweichende Kolonie zu Zoanthus sociatus zu stellen.

Zoanthus pulchellus (Duch. \& Mich.).

Fundort: Curaça o, Caracas-Bai, 5. Mai 1920 (2 Kolonien).

Von dieser Art liegen zwei leuchtend grün gefärbte Kolonien vor. DuERDEN (1898) beschreibt die Färbung des lebenden Tieres folgendermassen: "The proximal part of the column is pale buff in colour, the distal dark green or dark brown. Generally the disc is a bright green, with radiating lines equalling the tentacles in number. A common combination of colours is that of a green disc and brown peristome, and dark brown tentacles; in others the tentacles may be green or a light brown; again, in many the peristome is a bright pink, or, more rarely, yellow". Die Variabilität scheint also recht erheblich zu sein.

Sicher verbürgte Fundorte von Zoanthus pulchellus sind Columbien, Curaçao, Margarita, St. Thomas, Porto Rico, Jamaica und die Tortugas. Den Bermuda-Inseln fehlt die Species, wie ich schon früher (1910) betonte; denn Zoanthus dubius (Les.), mit dem VerriLl Zoanthus pulchellus (Duch. \& Mich.) identifiziert, muss als eigene Art aufgefasst werden.

Palythoa caribaeorum Duch. \& Mich.

(Taf. IX, Fig. 2).

Fundort: Cu raça o, Caracas-Bai, 5. Mai 1920 (2 Kolonien).

Am Strande von Curaçao kommt eine Form von Palythoa caribaeorum vor, die sich in mehrfacher Hinsicht von dem im Antillenmeer verbreiteten Typus unterscheidet. Während alle Exemplare, die ich bisher untersucht habe, in konserviertem Zustande gelblich oder hellbraun gefärbt waren, zeigen die beiden vorliegenden Kolonien eine bräunlich-rote Färbung. Doch ist darauf wohl wenig Gewicht zu legen, da nach Duerden (1898) Palythoa caribaeorum in Bezug auf die Färbung nicht unerheblich variiert, auch postmortale Veränderungen eine Rolle spielen können. „The colour differs”, schreibt Duerden, "but little from the yellow or cream of the previous species [Palythoa mammillosa], though colonies are sometimes brown, or may become so when taken from the water. In these the apices of the ridges may be white, and the tentacles and peripheral part of the disc dark brown". Die wichtigsten morphologischen Merkmale, durch die sich die Form von Curaçao von den früher (1910) von mir untersuchten Exemplaren von St. Thomas unterscheidet, sind in folgender Tabelle enthalten:

Rasse von St. Thomas.

Kolonie flach.

Alle Polypen einer Kolonie von gleicher Grösse

(5 $\mathrm{mm}$ im Durchmesser).

Zahl der Capitularfurchen 14-17. 28-34 Mesenterien.
Rasse von Curaçao.

Kolonie gewölbt.

Polypen einer Kolonie von ungleicher Grösse (Durchmesser zwischen 4,5 und $6 \mathrm{~mm}$ schwankend).

Zahl der Capitularfurchen 12-14. 26- 28 Mesenterien. 
.. Trotz dieser Verschiedenheiten glaube ich jedoch, die beiden von VAN DER HORST gesammelten Kolonien zu Palythoa caribaeorum stellen zu müssen, da sich im Bau des Sphinkters sowie in anderen anatomischen Merkmalen keine Unterschiede ergeben.

Die Art ist im Litoral der Antillen (Curaçao, St. Thomas, Porto Rico, Jamaica, Cuba) verbreitet.

Palythoa cáracasiana nov. spec.

(Taf. IX, Fig. 3).

Fundort: Cu raça o, Caracas-Bai, 25. Mai 1920 (4 Kolonien).

Palythoa caracasiana ist eine mit P. mammillosa offenbar nahe verwandte Art, die am Strande von Curaçao auf Korallenfels wenig umfangreiche Kolonien bildet. Der Durchmesser der grössten mir vorliegende Kolonie beträgt $54 \mathrm{~mm}$, ihre Höhe $18 \mathrm{~mm}$. Die Zahl der Polypen, welche sich am Aufbau

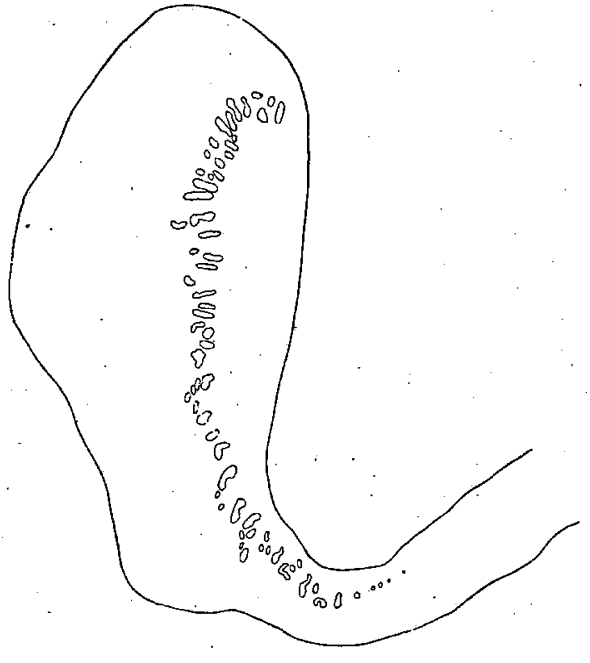

Fig. 13. Querschnitt durch den Sphinkter von Palythoa caracasiana. Die schwarze Umrisseiner Kolonie beteiligen, schwankt beträchtlich. An dem Material der Sammlung VAN DER HORST stellte ich die Zahl der Polypen auf 16, 18, 42 und 96 fest. Cönenchym und Polypen sind in konserviertem Zustande dunkelbraun. Der Umriss der Kolonien, die trotz ihrer starken Inkrustation mit Fremdkörpern nicht vollkommen starr, sondern biegsam sind, sich aber durch eine sehr erhebliche Zugfestigkeit auszeichnen, ist unregelmässig oval oder polygonal. Die Polypen erheben sich viel stärker als bei Palythoa mammillosa über die Fläche des Cönenchyms; gegeneinander sind sie wenig deutlich abgegrenzt. Der Durchmesser der Polypen beträgt 3-5 mm. Ausserordentlich charakteristisch für Palythoa caracasiana ist das Auftreten von 14 Capitularfurchen, die eine solche Tiefe aufweisen, wie ich sie kaum je bei einer Palythoa-Art gesehen habe. Von den Tentakeln und der Mundscheibe ist an meinem Untersuchungsmaterial infolge der starken Kontraktion der Tiere nichts zu erkennen. Das Schlundrohr weist eine histologisch sehr gut differenzierte Schlundrinne auf. Die Zahl der Mesenterien schwankt zwischen 28 und 32. Gonaden waren an keinem der von mir anatomisch untersuchten Polypen entwickelt. Die Inkrustation der Körperwand, die fast bis an den Sphinkter heranreicht, besteht zwar überwiegend aus kleinen Kalkstückchen, doch kommen auch hier und da Sandkörnchen vor. In mehreren Fällen fand ich auch, tief in das Innere der Mesoglöa eingebettet, vereinzelte Skelette von Radiolarien.

Der mesoglöale Sphinkter zeichnet sich durch eine beträchtliche Länge aus (Fig. 13). Seine beiden Enden nähern sich stark dem Entoderm, während die mittleren Teile in merklichem Abstande vom Entoderm verlaufen. Die Breite des Sphinkters wechselt etwas, doch kann man nicht sagen, dass sein distales Ende am breitesten sei. Eine starke Verschmälerung des Sphinkters findet nur an seinem proximalen Ende statt. An mehreren Stellen liegen 2-3 Muskelmaschen nebeneinander.

Schliesslich seien noch die wichtigsten differentialdiagnostischen Merkmale gegenüber Palythoa mammillosa in Form einer Tabelle zusammengesteltt:

\section{Palythoa mammillosa.}

Kolonie aus 10-- 250 Polypen bestehend. Durchmesser der Polypen 8-9 $\mathrm{mm}$.

18-20 Capitularfurchen.

Inkrustation der Körperwand überwiegend kieselig. 36-38 Mesenterien.

Sphinkter im grössten Teil seiner Ausdehnung nur aus einer Reihe von Muskelmaschen bestehend; Verschmälerung des proximalen Endes unbedeutend.

\section{Palythoa caracasiana.}

Kolonie aus 20-100 Polypen bestehend. Durchmesser der Polypen 3-5 mm. 14 Capitularfurchen. Inkrustation der Körperwand überwiegend kalkig. 28-32 Mesenterien.

Im grössten Teile des Sphinkters liegen mehrere Muskelmaschen nebeneinander; Auskeilung des Sphinkters am proximalen Ende kurz, aber sehr deutlich. 
Palythoa horstii nov. spec.

Fundort: Curaça o, Westpunt, 14. Mai $1920^{\circ}$ (2 Kolonien).

Als ich die beiden Kolonien von Palythoa horstii aus dem Amsterdamer Museum zugeschickt bekam, waren die Polypen kirschrot gefärbt, das Cönenchym war sandfarben. Nachdem die in Alkohol konservierten Tiere in meinem Arbeitszimmer eine Zeitlang dem diffusen Tageslicht ausgesetzt waren, verschwanden die leuchtenden Farben und machten einem unscheinbaren Graugelb Platz. Beide Kolonien sind auf Korallenkalk festgewachsen. Die eine Kolonie, welche vollkommen unversehrt ist, weist einen kreisrunden Umriss auf. Ihr Durchmesser beträgt $25 \mathrm{~mm}$, ihre Höhe $10 \mathrm{~mm}$. Die Basis, mit der die Kolonie dem Substrat aufsitzt, misst $15 \mathrm{~mm}$ im Durchmesser. Die Kolonie wird von 46 Polypen gebildet, die sich in kontrahiertem Zustande fast gar nicht über die Oberfläche des Cönenchyms erheben und gegeneinander kaum abgegrenzt erscheinen. Selbst bei Betrachtung mit der Lupe sieht man nichts anderes als die von 13 oder 14 Capitularfurchen umgebenen Mundöffnungen. Die zweite Kolonie, die wohl gewaltsam von der Unterlage losgerissen und hierbei beschädigt worden ist, scheint ursprünglich einen ovalen Umriss besessen zu haben. Sie unterscheidet sich von der ersten Kolonie dadurch, dass die Polypen auch im Zustande stärkster Kontraktion sich in Gestalt kleiner Buckel über die lẹicht gewellte Oberfläche des Cönenchyms erheben. Auch ist die gegenseitige Abgrenzung der Polypen deutlicher als bei der ersten Kolonie. Hier und da sind die Polypen sogar fünfeckig abgeplattet. Der Durchmesser der Polypen beträgt bei beiden Kolonien durchschnittlich $5 \mathrm{~mm}$.

Cönenchym und Polypen sind ausserordentlich stark inkrustiert. Neben Kalkkörnchen, die der Masse nach in dem Fremdkörperskelett von Palythoa horstii durchaus überwiegen, treten auch vereinzelt Kieselnadeln von Spongien und Foraminiferenskelette auf. Die Inkrustation erstreckt sich fast auf die ganze Mesoglöa. Nur ein ganz schmaler Streifen Bindegewebe auf der entodermalen Seite der Mesoglöa ist frei von kalkigen und kieseligen Fremdkörpern. In der Sphinkterregion der Körperwand reicht die Inkrustation bis unmittelbar an die Muskelmaschen des Ringmuskels heran. Zellinseln und Lakunen sind in der Mesoglöa reichlich vorhanden.

Infolge der starken Kontraktion des Untersuchungsmaterials war es mir nicht möglich, die Zahl der Tentakel mit Sicherheit festzustellen. Ein

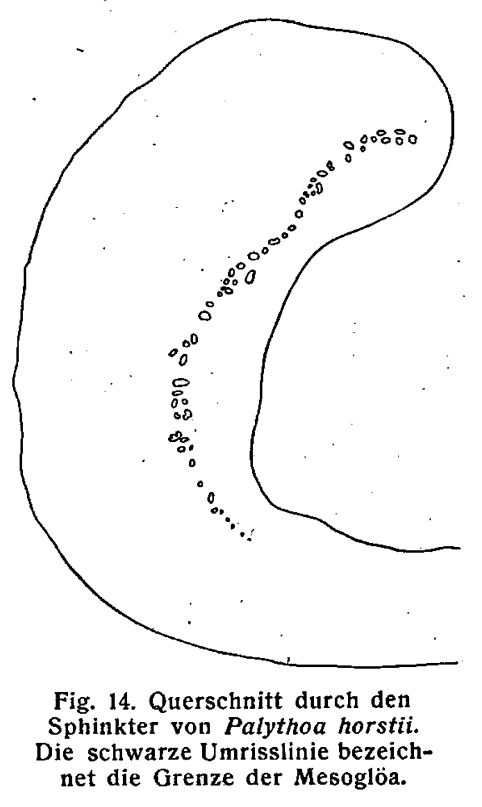
von mir untersuchter Polyp besass schätzungsweise 28 Tentakel. Das Schlundrohr, das sich durch den Besitz eines auffallend hohen Ektoderms auszeichnet, weist eine histologisch scharf differenzierte Siphonoglyphe auf. Die Zahl der Mesenterien betrug an einem von mir in Querschnitte zerlegten Individuum 30. Alle Mesenterien sind mit deutlichen Basalkanälen versehen.

Der Snhinkter ist kürzer als bei anderen Palythoa-Arten und nicht sehr kräftig entwickelt. Dies zeigt besonders deutlich ein Vergleich der Figuren 13 und 14. Beide Querschnitte sind mit Hilfe des Edingerschen Projektionsapparates bei gleicher Vergrösserung gezeichnet worden. Wir sehen, dass die Muskelmaschen im Sphinkter von Palythoa horstii sowohl an Zahl wie an Grösse hinter denjenigen von $P$. caracasiana zurückbleiben. Auch sind die Muskelmaschen von $P$. horstii nicht quer oval, sondern eher kreisförmig. Ausserordentlich charakteristisch ist die S-förmige Krümmung des Sphinkters, welche Figur 14 gut wiedergibt. Der Sphinkter verläuft also nicht in gleichem Abstande vom Entoderm, sondern nähert sich diesem an drei Stellen. Die Breite des Sphinkters schwankt ein wenig, indem in gewissen Regionen mehrere Muskelmaschen nebeneinander liegen. Die Auskeilung des proximalen Sphinkterendes ist deutlich.

Isaurus duchassaingi var. microtuberculata nov. var.

Fundorte: Curaçao, Caracas-Bai, 1. Mai 1920 (3 Exemplare).

Curaça o, Caracas-Bai, 6. Mai 1920 (66 Exemplare).

Isaurus duchassaingi kommt im Litoral von Curaçao in einer Varietät vor, die in biologischer und morphologischer Hinsicht sich von der in den westindischen Gewässern verbreiteten Form (DUERDEN 
1898 u. 1902, Pax 1910) unterscheidet. Ich schlage vor, sie als var. microtuberculata zu bezeichnen. Die konservierten Tiere erscheinen blass fleischfarben mit einem grauen Schimmer. Sie bilden auf Korallenfels festgewachsene Kolonien, an deren Aufbau sich 20 und mehr Individuen beteiligen. Um die im Folgenden mitgeteilten Messungen durchführen zu können, habe ich allerdings gerade die grössten Kolonien auseinanderbrechen müssen. Derartig individuenreiche Kolonien von Isaurus duchassaingi. habe ich bisher niemals aus Westindien erhalten. Ich kannte die Species bisher nur solitär lebend oder kleine Kolonien von 3 bis höchstens 8 Individuen bildend. Die beträchtliche Grösse der Kolonie scheint also ein biologisches Merkmal der am Strande von Curaçao heimischen Varietät zu sein. Sämtliche mir vorliegenden Exemplare sind kontrahiert. Obwohl die Art schon häufig in Westindien gesammelt worden ist, sind Stücke mit ausgebreiteter Mundscheibe und entfalteten Tentakeln wohl äusserst selten in die Hände eines Spezialisten gelangt. Auch DuERDEN (1902) schreibt in seinen "Actinians of Porto Rico": "The polyps are rarely seen in an expanded condition". Wie viele tropische Korallen scheint Isaurus duchassaingi nur des Nachts voll entfattet zu sein.

Da die Art schon wiederholt eingehend beschrieben worden ist, seien im Folgenden nur die Merkmale hervorgehoben, die für die var. microtuberculata charakteristisch erscheinen oder in denen das vorliegende Material mit den bisher veröffentlichten Beschreibungen nicht übereinstimmt. Um die Körpergestalt etwas genauer bestimmen zu können, habe ich eine Anzahl Messungen vorgenommen, die ich in Form einer Tabelle zusammengestellt habe. Um einen Massstab für den Betrag der Krümmung der Polypen zu erhalten, habe ich neben die Körperlänge, in der also die gesamte Krümmung mit inbegriffen ist, zum Vergleiche die Körperhöhe geschrieben, die nur die Länge des Lotes angibt, das man von der Spitze des Capitulum auf die Unterlage fällen kann. Je stärker gekrümmt ein Individuum ist, desto grösser ist natürlich auch die Differenz zwischen Körperlänge und Körperhöhe. Sämtliche Masse der folgenden Tabelle sind in Millimetern angegeben:

\begin{tabular}{|c|c|c|c|c|c|}
\hline $\begin{array}{c}\text { Basaler } \\
\text { Durchmesser. }\end{array}$ & $\begin{array}{c}\text { Apikaler } \\
\text { Durchmesser. }\end{array}$ & Körperlänge. & Körperhöhe. & $\begin{array}{l}\text { Länge des } \\
\text { tuberkelfreien } \\
\text { Abschnitts. }\end{array}$ & $\begin{array}{l}\text { Tuberkelregion } \\
\text { in } \% \text { der gesam- } \\
\text { ten Körperlänge. }\end{array}$ \\
\hline 4 & : 4 & 31 & 27 & 19 & 39 \\
\hline 7 & 5 & 54 & 32 & 21 & 61 \\
\hline 7 . & 5 & 35 & 25 & 22 & 37 \\
\hline 6 & 5 & 30 & 24 & 18 & 40 \\
\hline 12 & 6 & 46 & 28 & 34 & 26 \\
\hline 10 & 5 & 39 & 34 & 36 & 8 \\
\hline 9 & 7. & 22 & 20 & 14 & 36 \\
\hline 10 & 7. & 37 & 34 & 15 & 59 \\
\hline 11 & 6 & 40 & 27 & 25 & 33 \\
\hline 5 & 4 & 35 & 29 & 18 & 49 \\
\hline 8 & 5 & 32 & $10 \ldots$ & 5 & 84 \\
\hline 11 & 6 & 43 & 26 & 22 & 49 \\
\hline 10 & 6 & 43 & 22 & 24 & 44 \\
\hline 10 & 5 & 28 & 20 & 20 & 29 \\
\hline 7 & 5 & 27 & 25 & 7 & 74 \\
\hline 9 & 4 & 29 & 15 & 20 & 31 \\
\hline 4 & 2 & 10 & 10 & 10 & 0 \\
\hline 7 & 6 & 34 & 26 & 25 & 26 \\
\hline 11 & 7 & 37 & 32 & 20 & 46 \\
\hline 8 & 6 & 45 & 27 & 16 & 64 \\
\hline 7 & 5 & 26 & 25 & 10 & 61 \\
\hline 12 & 7 & 41 & 17 & 41 & 0 \\
\hline 12 & 5 & 38 & 25 & 28 & 26 \\
\hline 7 & 5 & 29 & 27 & 20 & 31 \\
\hline 8 & 6 & 30 & 23 & 15 & 50 \\
\hline 8 & 7 & 25 & 19 & 12 & 52 \\
\hline 10 & 8 & 36 & 20 & 22 & 39 \\
\hline 6 & 5 & 17 & 17 & 15 & 12 \\
\hline
\end{tabular}




\begin{tabular}{|c|c|c|c|c|c|}
\hline $\begin{array}{c}\text { Basaler } \\
\text { Durchmesser. }\end{array}$ & $\begin{array}{c}\text { Apikaler } \\
\text { Durchmesser. }\end{array}$ & Körperlänge. & Körperhöhe. & $\begin{array}{l}\text { Länge des } \\
\text { tuberkelfreien } \\
\text { Abschnitts. }\end{array}$ & $\begin{array}{l}\text { Tuberkelregion } \\
\text { in } \% \text { der gesam- } \\
\text { ten Körperlänge. }\end{array}$ \\
\hline 9 & 6 & 48 & 35 & 29 & $\cdots$ \\
\hline 11 & 8 & .54 & 26 & 54 & 0 \\
\hline 7 & 3 & 24 & 24 & 24 & 0 \\
\hline 8 & 7 & 27 & 25 & 20 & 26 \\
\hline 8 & 7 & 38 & 28 & 24 & 32 \\
\hline 6 & 5 & 30 & 25 & 16 & 47 \\
\hline 9 & 5 & 30 & 23 & 23 . & 23 \\
\hline$\cdot$ & 5 . & 27 & 24 & $\therefore 15$ & 44 \\
\hline 7 & 5 & 35 & 25 & 35 & 0 \\
\hline 8 & 5 & 36 & 32 & 30 & 17 \\
\hline 10 & 8 & 28 . & 25 & 28 & 0 \\
\hline 10 & 6 & 25 & 18 & 25 & 0 \\
\hline 7 & 7 & 35 & 28 & 35 & 0 \\
\hline 5 & 5 & 42 & 29 & 42 & 0 \\
\hline 10 & 7 & 39 & 36 & 33 & 15 \\
\hline 11 & 5 & 29 & 28 & 21 & $\therefore \quad 28$ \\
\hline 6 & 5 & 24 & 12 & 14 & , 42 \\
\hline 10 & 7 & 37 & 33 & 37 & 0 \\
\hline 9 & 5 & 27 & 22 & 27 & 0 \\
\hline 7 & 5 & 30 & 21 & 18 & 40 \\
\hline 8 & 6 & 29 & 23 & . 23 & 21 \\
\hline $11^{\circ}$ & 7 & 35 & 27 & 20 & 43 \\
\hline 8 &. & 44 & 34 & 34 & 23 \\
\hline$\cdot 6$ & 6 & 33 & 31 & 33 & 0 \\
\hline 10 & 10 & 33 & 30 & 19 & $\ldots 42$ \\
\hline 8 & 4 & 30 & 25 & 30 & $: 0$ \\
\hline 9 & 6 & 36 & 25 & 36 & 0 \\
\hline 8 & 7 & 42 & 30 & 42 & 0 \\
\hline
\end{tabular}

$\mathrm{Zu}$ den Messungen habe ich insgesamt 56 Individuen verwandt, die normal entwickelt und gut konserviert zu sein schienen. Jugendliche Exemplare und Kümmerformen blieben unberücksichtigt. Das Ergebnis der Messungen gestaltet sich folgendermassen:

\begin{tabular}{c|c|c|c|c|c|c}
\hline \hline & $\begin{array}{c}\text { Basaler } \\
\text { Durchmesser. }\end{array}$ & $\begin{array}{c}\text { Apikaler } \\
\text { Durchmesser. }\end{array}$ & Körperlänge. & Körperhöhe. & $\begin{array}{c}\text { Länge des } \\
\text { tuberkelfreien } \\
\text { Abschnitts. }\end{array}$ & $\begin{array}{c}\text { Tuberkelregion } \\
\text { in Prozenten der } \\
\text { gesamten } \\
\text { Körperlänge. }\end{array}$ \\
\hline Maximalwert. & 12 & 10 & 54 & 36 & 54 & 84 \\
\hline Minimalwert. & 4 & 2 & 10 & 10 & 5 & 0 \\
\hline $\begin{array}{c}\text { Durchschnitt sämt- } \\
\text { licher Messungen. }\end{array}$ & 8 & 6 & 32 & 25 & & 24 \\
\hline
\end{tabular}

Der basale Körperdurchmesser $(4-12 \mathrm{~mm})$ meines Untersuchungsmaterials stimmt recht gut überein mit den früher (1910) von mir an Stücken von Barbados festgestellten Massen (4-8 mm) und den von Duerden (1898) für Exemplare aus Jamaica angegebenen Werten $(4-10 \mathrm{~mm}$ ). Wie aus einem Vergleich des basalen und des apikalen Körperdurchmessers hervorgeht, ist der Körper von Isaurus duchassaingi nicht genau zylindrisch, sondern nach oben zu etwas verjüngt. Diese apikale Verjüngung 
zeigen $91 \%$ der Exemplare von Curaçao, nur $9 \%$ besitzen eine zylindrische Körperform. Unter dem Material aus Barbados befanden sich $70 \%$ zylindrische Polypen. Was die Körperlänge anlangt, so gibt DUERDEN (1898 u. 1902) für Exemplare von Jamaica und Porto Rico 22-42 mm an. Ich selbst fand (1910) an Tieren von Barbados 17-54 mm und an dem in Curaçao gesammelten Material 10-54 mm. Die mir vorliegenden Stücke sind ausserordentlich stark gekrümmt, bisweilen findet man Individuen,

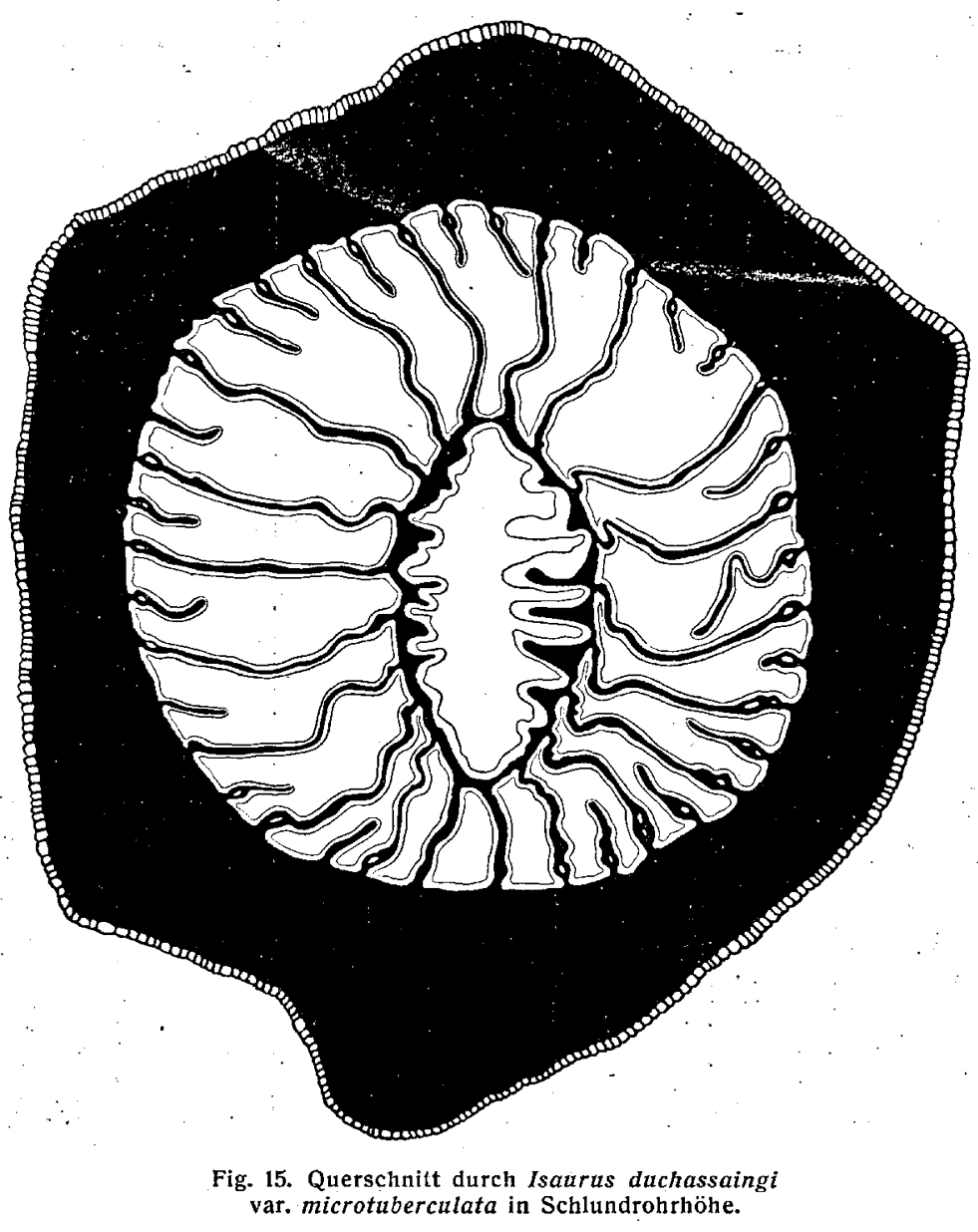
die nahezu rechtwinkelig gebogen sind. $96 \%$ des Materials von Curaçao sind gekrümmt, nur $4 \%$ sind gerade.

Die Tuberkel der Körperwand sind bei Isaurus duchassaingi in unregelmässigen Längsreihen angeordnet. Die westindischen Stücke, die ich früher (1910) untersucht habe, wiesen meist vier Längsreihen auf, die sich aus $6-8$ grossen Tuberkeln zusammensetzten. Dazwischen schoben sich häufig noch kürzere, meist nur aus 2-3 kleineren Tuberkeln bestehende Longitudinalreihen ein. Bei den in Curaçao gesammelten Exemplaren ist der Grundriss der einzelnen Tuberkel niemals kreisrund, sondern fast stets länglich-oval; die Höhe der Tuberkel ist sehr gering, und nicht selten verschmelzen die Tuberkel einer Longitudinalreihe zu schwachen Längsrippen. $15 \%$ meines Untersuchungsmaterials entbehrt der Tuberkelbildungen in der Körperwand. Meist besteht eine Längșreihe aus 4 einzelnen Tuberkeln; infolgedessen nimmt die Tuberkelregion durchschnittlich wenig mehr als ein Viertel der gesamten Körperlänge ein. In den extremsten Fällen waren $84 \%$ der Körperlänge mit Tuberkeln besetzt, doch findet sich nur bei $2 \%$ meines Untersuchungsmaterials eine derartige Ausdehnung der Tuberkelregion. Im histologischen Bau der Körperwand, insbesondere der Differenzierung des Ektoderms, stimmt die var. microtuberculata mit der typischen Form duchassaingi überein. Was die Zahl der Mesenterien anlangt, so finden sich in der Literatur darüber folgende Angaben:

\begin{tabular}{|c|c|c|}
\hline $\begin{array}{c}\text { Zahl der } \\
\text { Mesenterien. }\end{array}$ & $\begin{array}{c}\text { Herkunft des } \\
\text { Untersuchungsmaterials. }\end{array}$ & Autor. \\
\hline $\begin{array}{l}36-46 \\
38 \\
40 \\
40\end{array}$ & $\begin{array}{l}\text { Bahama-Inseln } \\
\text { St. Thomas, Barbados } \\
\text { Porto Rico } \\
\text { Bermuda-Inseln }\end{array}$ & $\begin{array}{c}\text { MC MURRICH } 1896 \\
\text { PAX } 1910 \\
\text { DUERDEN } 1902 \\
\text { VERRILL } 1907\end{array}$ \\
\hline
\end{tabular}

Die in Curaçao gesammelten Tiere weisen, soweit ich sie anatomisch untersucht habe, ausnahmslos 40 Mesenterien auf. Figur 15 zeigt einen. Querschnitt durch eines der grössten Exemplare der Sammlung vaN DER HORST in Schlundrohrhöhe. Man sieht in der Mitte das gefurchte Schlundrohr mit der kaum differenzierten Schlundrinne und die 40 radiärgestellten, mit einem dünnen Entodermbelag überzogenen, zum Teil mit nur winzigen Basalkanälen ausgestatten Mesenterien, darunter das dorsale unvollständige und das ventrale vollständige Richtungsmesenterienpaar. Die äussere Umgrenzung des Querschnitts bildet die durch ihre Dicke bemerkenswerte Körperwand, deren kantiger Umriss 
durch die zapfenförmig vorspringenden Tuberkel bedingt wird. Das Ektoderm, dessen Struktur schon früher eingehend beschrieben worden ist (DUERDEN 1898 u. 1902, PAX 1910), erreicht etwa nur ein Zehntel der Dicke der Mesoglöa. Ein unterhalb des Schlundrohrs gelegter Schnitt (Fig. 16) zeigt, dass die Körperwand im proximalen Teile der Polypen noch eine grössere Mächtigkeit besitzt als im distalen Abschnitt. Nur die vollständigen Mesenterien sind mit Filamenten ausgestattet. Im basalen Teile der Polypen verbreitern sich die Mesenterien, treten dicht aneinander und bringen auf diese Weise ein Art Verschluss der Gastrovaskularhöhle zu stande. So kommt es zur Differenzierung eines anscheinend soliden Stieles. Ein durch den „Stiel” gelegter Querschnitt (Fig. 17) zeigt, dass er aus einem Netzwerk
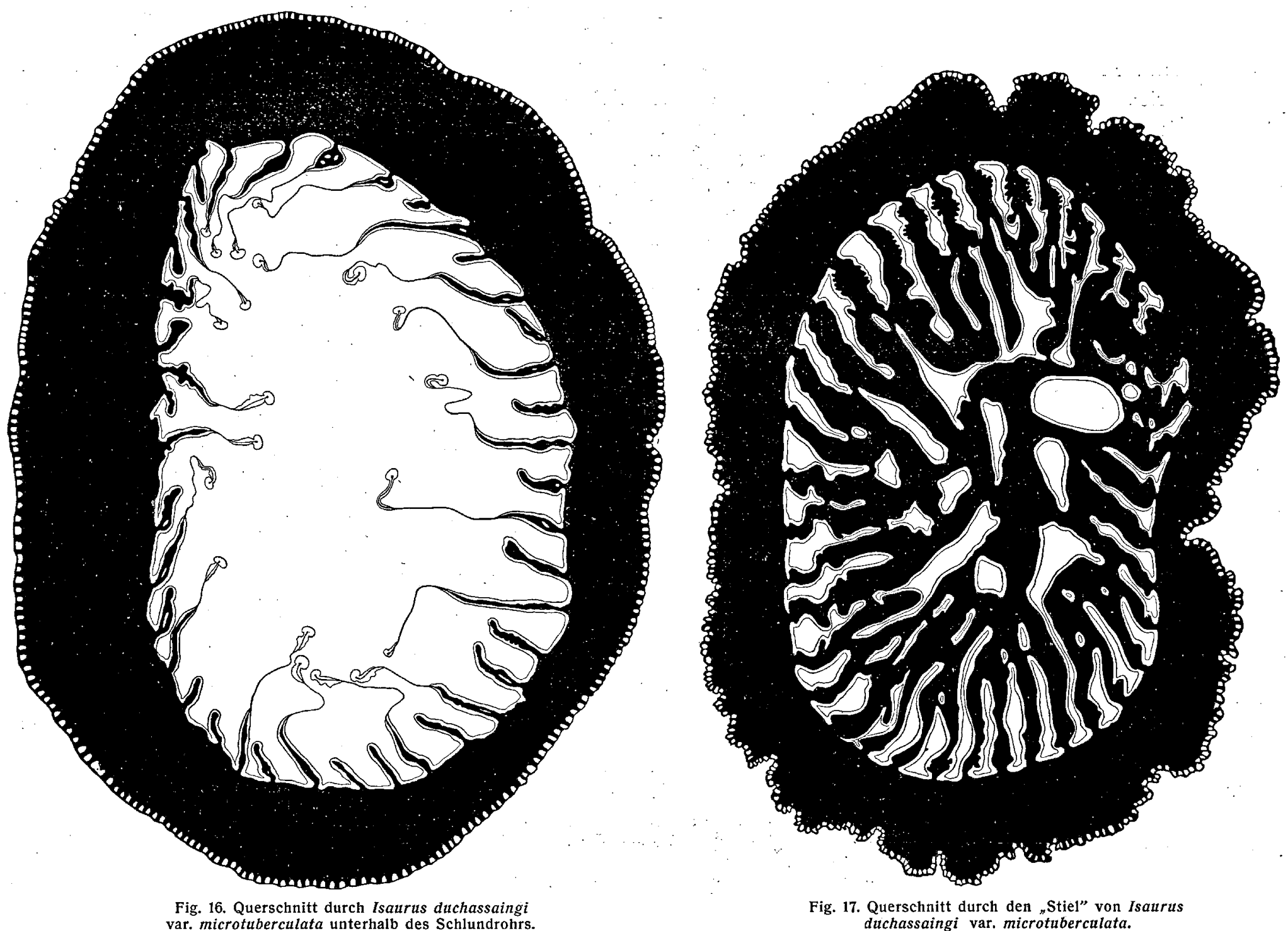

mesoglöaler Balken besteht, die im Zentrum zusammenfliessen. Zwischen den Mesoglöabalken befinden sich zahlreiche spaltförmige Hohlräume von unregelmässiger Gestalt.

Der Sphinkter der var. microtuberculata stimmt mit demjenigen von Isaurus duchassaingi durchaus überein; sein distaler Abschnitt (Fig. 20) setzt sich aus einer grossen Anzahl dicht liegender, etwa ovaler Maschen zusammen und unterscheidet sich wesentlich von dem Sphinkter des im Folgenden beschriebenen Isaurus gelatinosus. Alle von mir untersuchten Exemplare waren steril. Fertile Stücke von Isaurus duchassaingi sind wohl bisher überhaupt noch nicht beobachtet worden; wenigstens wird in der Literatur das Fehlen von Gonaden mehrfach hervorgehoben (DuERDEN 1898, 1902, PAX 1910). 
Isaurus gelatinosus nov. spec.

Fundort: Curaça o, Caracas-Bai, Mai 1920 (2 Kolonien).

Vergesellschaftet mit Zoanthus sociatus lebt in Curaçao auf Kalkblöcken des Strandes eine zweite Isaurus-Art, die äusserlich etwas an die in den westindischen Gewässern verbreitete Palythoa grandis erinnert, sich aber schon durch das Fehlen von Inkrustationen der Körperwand von ihr unterscheidet. Von den beiden mir vorliegenden Kolonien enthält die eine 18, die andere 38 Individuen. Die Farbe der Polypen und des Cönenchyms ist in konserviertem Zustande hellbraun. Das Cönenchym ist ausserordentlich dürftig entwickelt. Dadurch, dass die Polypen meistens gruppenweise von einem Punkte entspringen, erübrigt sich die Ausbildung besonderer Stolonen, und das Cönenchym tritt uns eigentlich nur in Gestalt lamellöser Verbreiterungen der Polypenbasis entgegen. Die Gestalt der Polypen ist etwa zylindrisch; einige Exemplare zeigen eine sehr deutliche Gliederung in Scapus und Capitulum, bei anderen ist die Grenze beider Körperregionen verwischt. Die für Isaurus duchassaingi und andere Arten der Gattung charakteristische Krümmung der Polypen ist bei I. gelatinosus nur schwach angedeutet, Tuberkel der Körperwand fehlen fast vollständig. Jedenfalls sind sie noch schwächer entwickelt als bei der oben beschriebenen var. microtuberculata des I. duchassaingi. Wo sie überhaupt nachweisbar sind, nehmen sie weniger als $10 \%$ der gesamten Körperlänge ein und sind nur bei sorgfältigster Untersuchung des Materials zu erkennen.

An 14 besonders gut konservierten Exemplaren nahm ich folgende Masse:

\begin{tabular}{c|c|c|c}
\hline $\begin{array}{c}\text { Basaler } \\
\text { Durchmesser. }\end{array}$ & $\begin{array}{c}\text { Apikaler } \\
\text { Durchmesser. }\end{array}$ & Körperlänge. & Körperhöhe. \\
\hline 18 & 7 & 35 & 35 \\
11 & 10 & 38 & 28 \\
8 & 5 & 20 & 18 \\
14 & 9 & 41 & 22 \\
14 & 8 & 24 & 20 \\
14 & 9 & 56 & 50 \\
8 & 6 & 42 & 35 \\
12 & 9 & 20 & 17 \\
12 & 5 & 28 & 25 \\
7 & 8 & 42 & 40 \\
9 & 5 & 34 & 30 \\
8 & 6 & 36 & 31 \\
8 & 6 & 45 & 42 \\
5 & 4 & 33 & 30 \\
& & & \\
\hline
\end{tabular}

Das Ergebnis der Messungen gestaltet sich folgendermassen:

\begin{tabular}{c|c|c|c|c}
\hline & $\begin{array}{c}\text { Basaler } \\
\text { Durchmesser. }\end{array}$ & $\begin{array}{c}\text { Apikaler } \\
\text { Durchmesser. }\end{array}$ & Körperlänge. & Körperhöhe. \\
\hline Maximalwert. & 18 & 10 & 56 & 50 \\
\hline Minimalwert. & 5 & 4 & 20 & 17 \\
\hline $\begin{array}{c}\text { Durchschnitt sämt- } \\
\text { licher Messungen. }\end{array}$ & 11 & 7 & 35 & 30 \\
\hline
\end{tabular}

Wie gering die Krümmung der Polypen ist, geht deutlich aus der kleinen Differenz von Körperlänge und Körperhöhe hervor, die höchstens 6, mindestens 3 und durchschnittlich $5 \mathrm{~mm}$ beträgt. Für 
Isaurus duchassaingi var. microtuberculata hatten wir als entsprechende Werte 18, 0 und $7 \mathrm{~mm}$. gefunden (S. 113). Das Capitulum ist mit ganz feinen, nur bei Lupenbetrachtung wạhrnehmbaren Radiär-



Fig. 19. Querschnitt durch das Schlundrohr eines geschlechtsreifen Polypen von Isaurus gelatinosus. Das Schlundrohr ist. spaltförmig und furchenlos.

streifen versehen, die so flach sind, dass sie kaum den Namen Furchen verdienen. Von den Organen des oralen Körperpols ist wegen der starken Kontraktion der Tiere bei äusserer Betrachtung nichts zu sehen. Die Tentakel sind kurz und ausgesprochen randständig. Ihre Zahl liess sich an meinem Untersuchungsmaterial nicht mit Sicherheit feststellen. Das Schlundrohr der jüngeren Polypen ist im Querschnitt annähernd oval (Fig. 18) und mit 14 Längsfurchen versehen, die aber nur Einstülpungen des verhältnismässig hohen Schlundrohrektoderms darstellen. Polsterbildungen der Mesoglöa kommen nicht vor. Die älteren Polypen besitzen ein spaltförmiges Schlundrohr (Fig. 19) ohne Längsfurchen und mit einem verhältnismässig niedrigen $\mathrm{Ek}$ toderm, das aber immerhin das Entoderm an Mächtigkeit übertrifft. Die Gesamtzahl der Mesenterien war bei Polypen der verschiedensten Altersklassen die gleiche, nämlich 42 . Eines der von mir untersuchten Exemplare

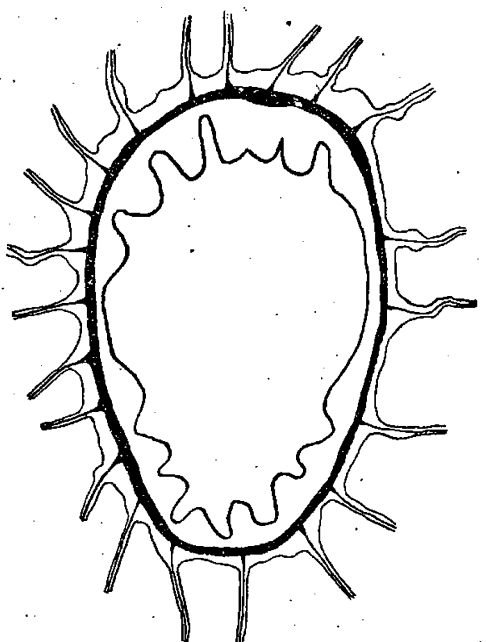

Fig. 18. Querschnitt durch das Schlundrohr eines jungen Polypen von Isauruts
gelatinosus. Das Schlundrohr ist oval und mit Längsfurchen versehen.

war fertil. In der Gonadenregion der Mesenterien fanden sich zahlreiche Eier mit einem grossen bläschenförmigen Kern und einem deutlich hervortretenden Nucleolus.

Der Sphinkter von Isaurus gelatinosus (Fig. 21) ist vor allem dadurch charakterisiert, dass sein distaler Teil sich aus verhältnismässig grossen Maschen zusammensetzt, die durch beträchtliche $Z$ wischenräume voneinander getrennt werden. Ein Vergleich der beiden Figuren 20

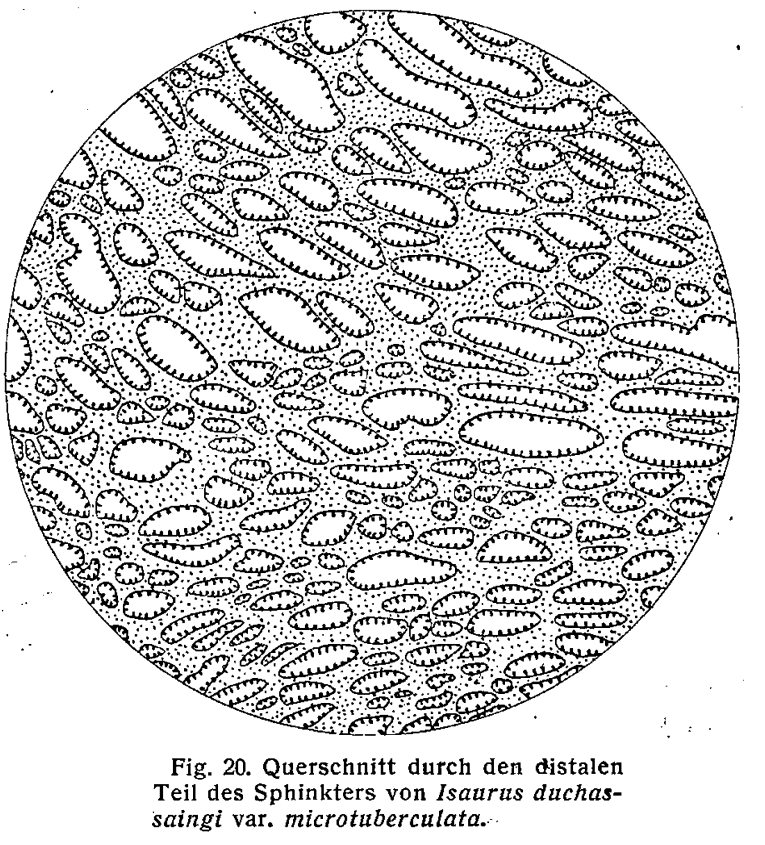

und 21, die bei gleicher Vergrösserung mit Hilfe des Projektionsapparates gezeichnet worden sind, 
lässt deutlich den Unterschied zwischen Isaurus duchassaingi und I. gelatinosus hervortreten. Der Sphinkter von Isaurus gelatinosus besitzt, besonders im distalen Teile, weniger, aber grössere Maschen

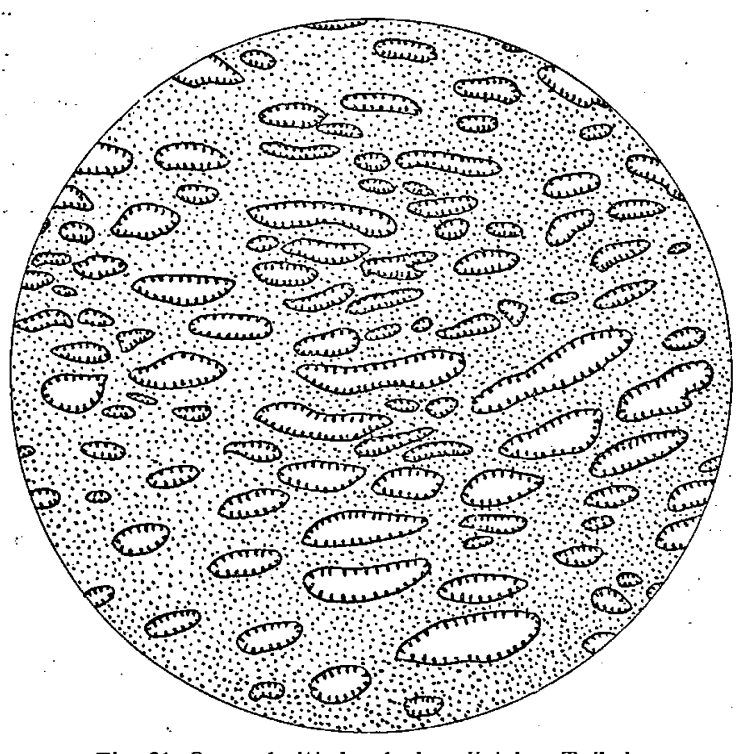

Fig. 21. Querschnitt durch den distalen Teil des Sphinkters von Isaurus gelatinosus. als derjenige von $I$. duchassaingi. Im proximalen Teile tritt dieser Unterschied weniger deutlich hervor.

\section{Ceriantharia.}

Ceriantheopsis - spec.

(Taf. IX, Fig. 9 u. 12).

Fundorte: Cu raça o, Caracas-Bai, 3. Mai 1920 (7Exemplare). Cu ra ç a o, Caracas-Bai, 18. Mai 1920 (4 Exemplare). Cu ra ça o, Spaansche Haven, 6. Mai 1920 (1Exemplar).

Cu raça o, Spaansche Haven, 20. Mai 1920(2 Exemplare).

Die Ausbeute VAN DER HORSTs aus Curaçao enthält eine Reihe junger Cerianthiden, die noch nicht geschlechtsreif sind und noch nicht lange ihre Wohnröhren bezogen haben können. Ihre sichere Bestimmung wäre nur möglich, wenn auch erwachsene Tiere vorlägen oder wenigstens gleichalterige Individuen von den aus Westindien beschriebenen Arten zu Gebote ständen. Um eine spätere Identifizierung aber zu ermöglichen, habe ich ein junges und ein etwas älteres Tier photographiert (Taf. IX, Fig. 9 und12) und einen Querschnitt durch das kleinere der beiden Stücke abgebildet (Fig. 22).

Aus Westindien und seinen Nachbargebieten sind bisher 3 Cerianthiden bekannt geworden: Ceriantheopsis americanus Verr., der an der Ostküste der Vereinigten Staaten von Cape Cod bis Florida verbreitet ist und dessen Südgrenze an den Tortugas und den Marquesas Cays (PAX 1910) liegt, eine zweite ihm äusserlich ähnliche Cerianthide, die Duerden (1902) ausPorto Rico erwähnt, und schliesslich der bisher anatomisch nicht untersuchte Cerianthus natans Verr. der Bermuda-Inseln.

Die Cerianthide, die von der Challenger-Expedition an der Mündung des Rio de la Plata gefunden und

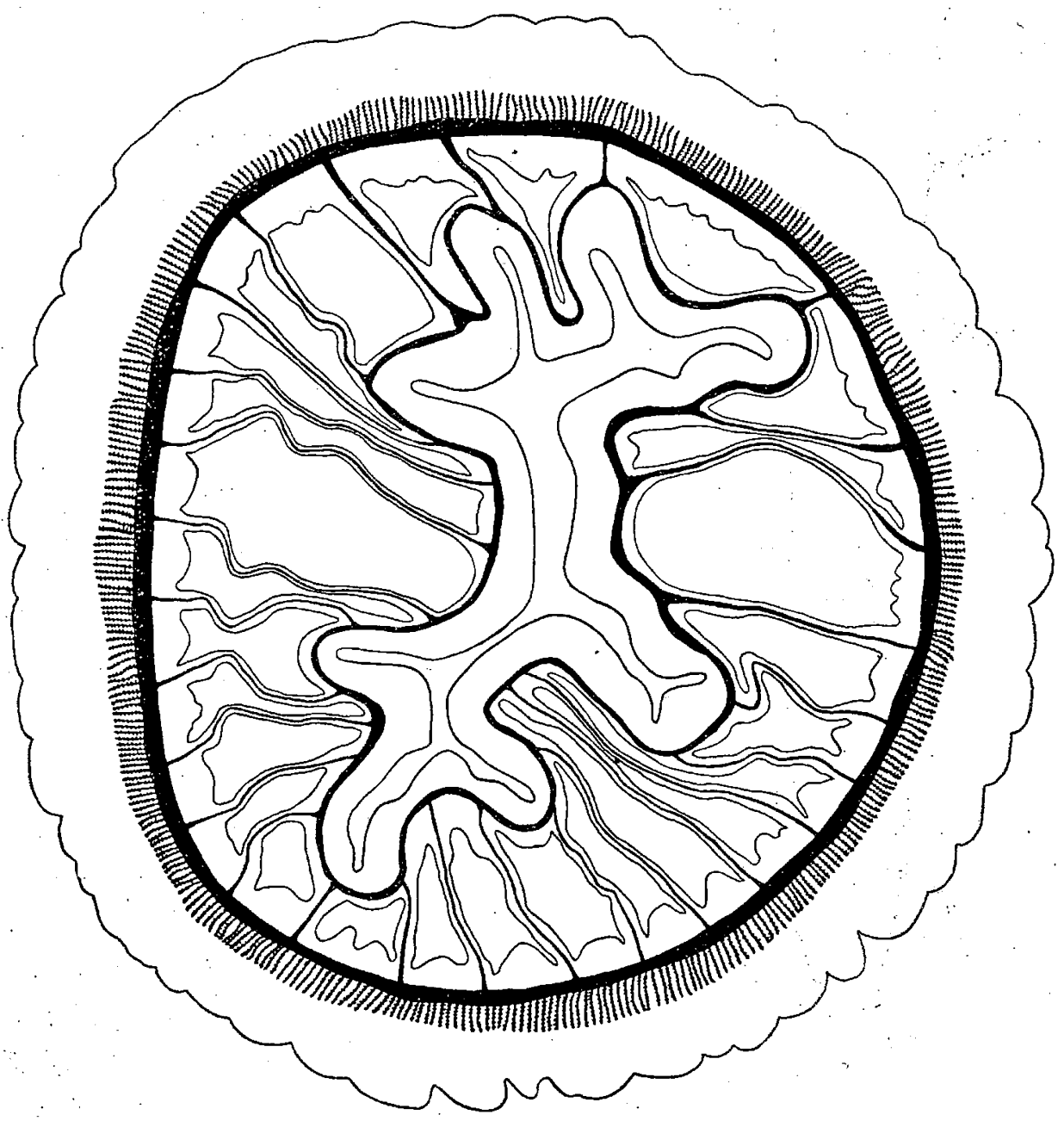

Fig. 22. Querschnitt durch Ceriantheopsis spec. in Schlundrohrhöhe.

mit C. americanus identifiziert wurde, dürfte, wie ich schon früher (1910) betont habe, wohl sicher davon zu trennen sein. 


\section{Die tiergeographischen Beziehungen der Actiniarien- und Zoantharienfauna von Curaçao.}

Als ich 1910 meine „Studien an westindischen Aktinien” veröffentlichte, war ich, ohne es in dieser Arbeit allerdings auszusprechen, der Meinung, dass die Erforschung der westindischen Seeanemonen nunmehr zu einem gewissen Abschlusse gelangt sei und spätere Studien die Zahl der aus dem amerikanischen Mittelmeer bekannten Arten nicht wesentlich vermehren würden. Die neueste Zeit hat meiner Auffassung nicht recht gegeben. 1922 beschrieb WaTzL von den Bahama-Insein 8 neue Actiniarien, und in der vorliegenden Mitteilung habe ich selbst 5 Aktinienspezies und 3 Zoantharienarten sowie eine Zoantharienvarietät neu aufgestellt. Unter diesen Umständen erscheint mir die Frage, ob die von VAN DER HORST in Curaçao neu aufgefundenen Arten Endemismen darstellen, verfrüht. Sehr wahrscheinlich ist eine derartige Annahme nach allem, was wir über die Verbreitung der Actiniarien, Zoantharien und Ceriantharien wissen, nicht. Vielmehr darf man wohl erwarten, dass die bis jetzt nur aus Curaçao nachgewiesenen Spezies später auch in anderen Teilen des Antillenmeeres aufgefunden werden.

Über die Verbreitung der einzelnen Arten gibt folgende Tabelle Aufschluss:

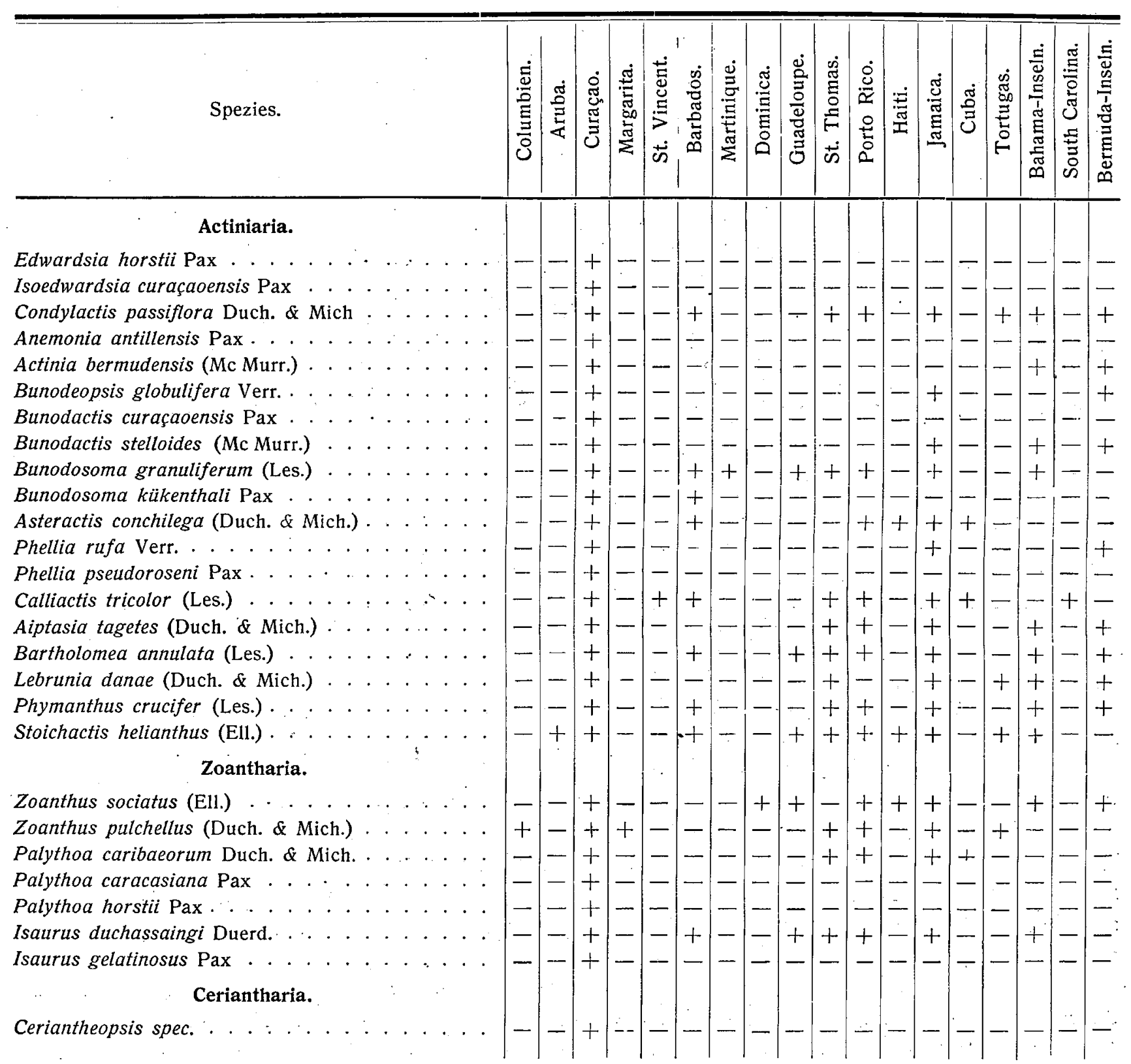


Keine der von van DER HORST in Curaçało gesammelten Actiniarien, Zoantharien und Ceriantharien kommt ausserhalb des westindischen Faunenbezirks vor, dessen nördlichste Ausläufer.noch die Bermuda-Inseln umfassen. So bestätigt diese Sammlung unsere bisherigen Erfahrungen, denen zufolge sich Westindien als ein faunistisch scharf abgegrenztes Gebiet von grosser Selbständigkeit erweist. Wenn wir von den von mir als neu beschriebenen Arten absehen, können wir unter den Actiniarien und Zoantharien zwei Gruppen unterscheiden: solche, die auch die Bermudas besiedelt haben, und solche, die diesen äussersten Vorposten der westindischen Fauna nicht mehr erreicht haben. Zu der ersten Gruppe gehören aus der Fauna von Curaçao Condylactis passiflora, Actinia bermudensis, Bunodeopsis globulifera, Bunodactis stelloides, Phellia rufa, Aiptasia tagetes, Bartholomea annulata, Lebrunia danae, Phymanthus crucifer und Zoanthus sociatus. Vertreter der zweiten Gruppe sind Bunodosoma granuliferum, Bunodosoma kükenthali, Asteractis conchilega, Calliactis tricolor, Stoichactis helianthus, Zoanthus pulchellus, Palythoa caribaeorum und Isaurus duchassaingi. Die Fauna der Bermuda-Inseln ist eine verarmte westindische Fauna.

Was die Verbreitung der Actiniarien und Zoantharien innerhalb des amerikanischen Mittelmeeres anlangt, so liefert die Sammlung VAN DER HORST-wichtige Belege für die Anschauung von der



Karte 1. Geographische Verbreitung von Condylactis passiflora $\bigcirc$; Stoichactis helianthus $\bigcirc$ und Phymanthus crucifer + .

Gleichförmigkeit der Antillenfauna. Von den 19 Actiniarien, die nunmehr aus Curaçao nachgewiesen sind, waren schon früher 12 aus Jamaica, 9 von den Bahama-Inseln und je 8 von Barbados, St. Thomas und Porto Rico bekannt, und unter den 7 Zoantharien von Curaçao finden wir je 4 in Jamaica und Porto Rico wieder. Die scheinbar vorhandenen Differenzen in der Zusammensetzung der Aktinienfauna der. Antillen beruhen gewiss zum guten Teile lediglich auf der Lückenhaftigkeit unserer Kenntnisse; hier und da mögen sie auch ökologisch bedingt sein. Die Mangrove bevölkert eine wesentlich andere Fauna als etwa den Aussenrand eines Korallenriffs. Grössere Unterschiede aber, als sie die Seeanemonen der Caracas-Bai und des Spaansch Water in Curaçao zeigen, finden sich auch nicht unter der Aktinienbevölkerung der Antillen.

Betrachten wir die Verteilung einzelner Arten an der Hand der Karten I und II, so tritt uns die gleichförmige Besiedlung der Antillen noch deutlicher vor Augen, gleichzeitig aber auch die offenkundige Abhängigkeit der geographischen Verbreitung der Seeanemonen von den Meeresströmungen, deren 
Verlauf auf den beiden Karten durch schwarze Pfeile angegeben wird '). Leider ist das Bereich der Yucatanströmung und das Ursprungsgebiet des Floridastroms in Bezug auf seine Actiniarien und Zoantharien noch vollkommen unerforscht, wie ja auch das Material zur Lösung der tiergeographischen



Die Verbreitung von Bunodeopsis globulifera deckt sich vollkommen mit derjenigen von Phellia rufa.

Probleme, die sich an die Frage nach den Beziehungen der westindischen Aktinienfauna zu derjenigen der pazifischen Küste Mittelamerikas knüpfen (VERRILl 1869, DuERDEN 1898), noch überaus dürftig ist. Hier bleibt für künftige Forschungen noch viel zu tun übrig.

Bresla u, 30. März 1924 .

1) Bezüglich der Meeresströmungen verweise ich auf die Karte\} von HANS H. F. MEYER, Atlantischer Ozean. Wasserbewegung an der Oberfläche im Februar in winkeltreuer azimutaler Äquatorprojektion (Massstab 1:30000 000). in: Veröffentl. Inst. f. Meereskunde, Reihe A, Heft 11 Kartenausgabe, 1923. 


\section{VERZEICHNIS DER BENÜTZTEN LITERATUR.}

1817. LesUeUR, C. A.: Observations on several species of the genus Actinia. Journ. Acad. nat. science Philadelphia. Vol. 1. 1850. Duchassaing, P.: Animaux radiaires des Antilles. Paris.

1860. Duchassaing, P. et J. MichelotTı: Mémoire sur les Coralliaires des Antilles. Mem. Accad. Torino, ser. 2, Vol.19. 1864. VerRill, A. E.: Revision of the Polypi of the eastern coast of the United States. Mem. Boston Soc. nat. Hist.Vol.1.

1869. VerRill, A. E.: Comparison of the Coral Faunae of the Atlantic and Pacific Coasts of the Isthmus of Darien. Amer. Natural. Vol. 3.

1883. Andres, A.: Le Attinie. Atti R. Accad. Lincei, ser. 3, Vol. 14.

1889. MC MURRich, J. P.: A contribution to the Actinology of the Bermudas. Proc. Acad. nat. scienc. Philadelphia.

1889. MC MURRICH, J. P.: The Actiniaria of the Bahama Islands. Journ. Morphol. Vol. 3.

1893. MC MURrich, J. P.: Report on the Actiniae collected by the United States Fish Commission Steamer ,Albatross'. Proc. U.S. Nation. Mus. Vol. 16.

1896. Duerden, J. E.: Zoanthidae at Port Henderson. Journ. Inst. Jamaica Vol. 2.

1896. MC MURRICH, J. P.: Notes on some Actinians from the Bahama Islands, collected by the late Dr. J. I. Northrop. Ann. New York Acad. Science. Vol. 9.

1897. Duerden, J. E.: The Actiniarian family Aliciidae. Ann. Mag. Nat. Hist. ser. 6, Vol. 20.

1898. Duerden, J. E.: Jamaican Actiniaria. Part I. Zoantheae. Transact. Roy. Dublin Soc. ser. 2, Vol. 6.

1898. Duerden, J. E.: The Actiniaria around Jamaica. Journ. Inst. Jamaica Vol. 2.

1898. DuERDEN, J. E.: The geographical distribution of the Actiniaria of Jamaica. Natural Science Vol. 12

1898. MC MURRICH, J. P.: Report on the Actiniaria collected by the Bahama Expedition of the State University of Iowa 1893. Bull. Laborat. nat. Hist. 1898.

1899. VerRiLl, A. E.: Description of imperfectly known and new Actinians, with critical notes on other species. Part II-V. Americ. Journ. scienc. ser. 4, Vol. 7.

1900. Duerden, J. E.: Jamaican Actiniaria. Part II. Stichodactylinae and Zoantheae. Transact. Roy. Dublin Soc. ser. 2, Vol. 7.

1900. PARKeR, G. H.: Synopses of North American Invertebrates. Part XIII. The Actiniaria. Americ. Naturalist Vol. 34.

1900. Verrill, A. E.: Additions to the Anthozoa and Hydrozoa of the Bermudas. Transact. Connecticut Acad. Vol. 10.

1901. Verrill, A. E.: Additions to the Fauna of the Bermudas from the Yale Expedition of 1901. Transact. Connecticut. Acad. Vol. 11 .

1902. Duerden, J. E.: On the Actinian Bunodeopsis globulifera Verrill. Transact. Linn. Soc. ser. 2, Zool. Vol. 8.

1902. Duerden, J. E.: Report on the Actinians of Porto Rico. Bull. U.S. Fish. Comm. f. 1900, Vol. 2.

1903. Duerden, J. E.: West Indian Sponge-incrusting Actinians. Bull. Amer. Mus. Nat. Hist. Vol. 19.

1905. MC MURRICH, J. P.: A revision on the Duchassaing and Michelotti Actinian types in the Museum of Natural History, Turin. Boll. Mus. Zool. Anat. comp. Univ. Torino Vol. 20.

1907. Verrill, A. E.: The Bermuda Islands. Part V. An account of the Coral reefs. Transact. Connecticut Acad. Vol.12.

1908. TUR, J.: Rozdwojenie czulika u ukwialu Anemonia sulcata. Spraw. Tow. nauk. Warszawsk., wydz. nauk. matem. przyrodn.

1910. CARY, L. R.: The formation of germ layers in Actinia bermudensis. Biol. Bull. Woods Hole Vol. 19.

1910. PAX, F.: Diagnosen neuer westindischer Aktinien. Zool. Anzeig. Vol. 36.

1910. PaX, F.: Studien an westindischen Actinien. Zoolog. Jahrb. Suppl. Vol. 11.

1911. HaRGitT, C. W.: Cradactis variabilis, an apparently new Tortugan Actinian. Papers Tortugas Laborat. Vol. 3.

1911. MC CLEnDon, J. F.: On adaptations in structure and habits of some marine animals of Tortugas, Florida. II. On adaptations of the reef anemone Cradactis variabilis. Papers Tortugas Laborat. Vol. 3.

1913. Hausding, B.: Studien über Actinoloba dianthus. Arch. Entwicklungsmech. Vol. 38.

1914. PAX, F.: Die Actinien. Ergebn. ut. Fortschr. Zool. Vol. 4.

1920-21: STEPHENSON, T. A.: On the classification of Actiniaria I-III. Quart. Journ. microscop. science. Vol.64-65.

1921. Fulton, J. F.: Concerning the vitality of Actinia bermudensis, a study in symbiosis. Journ. exper. Zool. Vol. 33.

1922. Watzl, O.: Die Actiniarien der Bahamainseln auf Grund der Sammlung des Herrn Dr. N. Rosén (1908-09) Arkiv zoologi, Vol. 14.

1924. PAX, F.: Anthozoen des Leidener Museums. Zoolog. Mededeel. Leiden, Deel VIII. 


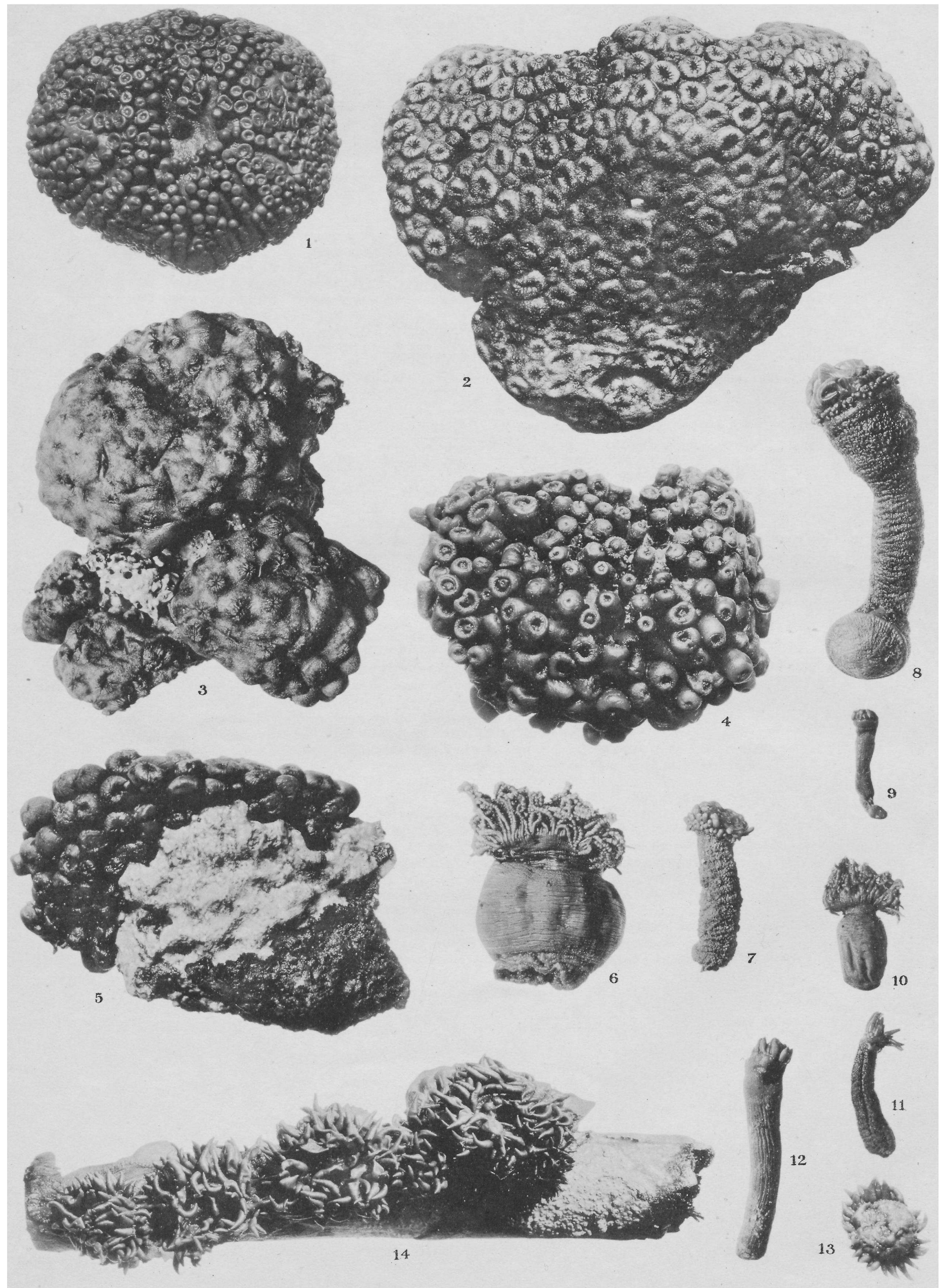

F. Pax und L. Pohl phot. 
TAFEL IX

\section{Charaktertiere des Strandes von Curaçao.}

Fig. 1. Stoichactis helianthus (E11.), kleinwüchsige Form, von oben gesehen.

Fig. 2. Palythoa caribaeortum Duch. \& Mich., in einer für das Litoral von Curaçao charakteristischen Rasse.

Fig. 3. Palythoa caracasiana nov. spec., Habitusbild.

Fig. 4. Kolonie von Zoanthus sociatus (Ell.), das typische lockere Wachstum der Art zeigend.

Fig. 5. Kolonie von Zoanthus sociatus (Ell.); gedrungene Wuchsform.

Fig. 6. Bartholomea annulata (Les.) mit rosenkranzförmigen Tentakeln; Seitenansicht.

Fig. 7. Phellia pseudoroseni nov. spec., Seitenansicht.

Fig. 8. Phellia rufa Verr., Habitusbild.

Fig. 9. Ceriantheopsis spec., junges Tier.

Fig. 10. Bartholomea annulata (Les.), junges Tier.

Fig. 11. Edwardsia horstii nov. spec., deutlich die Gliederung der Körperwand in Scapus und Capitulum zeigend.

Fig. 12. Ceriantheopsis spec., älteres, aber noch nicht geschlechtsreifes Tier.

Fig. 13. Jugendform von Asteractis conchilega (Duch. \& Mich.) mit schmalem Randkragen, aber kräftig entwickelten Saugwarzen.

Fig. 14. Stück einer Mangrovewurzel mit zahlreichen Exemplaren von Aiptasia tagetes (Duch. \& Mich.) besetzt. 